\title{
Unveiling Harriet Backer: ICP-OES study for the characterisation of the colour tubes from her original paint box
}

\author{
Francesco Caruso ${ }^{1} \mathbb{D}$, Sara Mantellato ${ }^{2^{*}}\left(\mathbb{D}\right.$, Noëlle L. W. Streeton ${ }^{1}\left(\mathbb{D}\right.$ ) and Tine Frøysaker ${ }^{1} \mathbb{0}$
}

\begin{abstract}
Micro-samples from 57 original paint tubes used between 1904 and 1909 by the Norwegian painter, Harriet Backer were studied with inductively coupled plasma-optical emission spectroscopy (ICP-OES). This accurate elemental characterisation of Backer's original materials aims to fill several gaps in conservation science, painting conservation, and art technology. Firstly, it provides a novel and validated analytical method that can be applied to other painting materials. Secondly, ICP-OES offers scientific information about different oil colours from the late 19th to the early 20th century by Dr. Schoenfeld \& Co., Düsseldorf, currently known as Lukas-Nerchau, whose archives were depleted during the Second World War. This work also lends new insights into the paintings of a renowned female Norwegian artist, whose painting materials have been little studied to date. ICP-OES results, coupled with a comprehensive, illustrated catalogue of paint tubes, will be instrumental for ongoing scientific investigations of this painter's body of work.
\end{abstract}

Keywords: Norway, Uvdal, Christiania, Dr. Schoenfeld, Lukas-Nerchau, Analysis, Composition, PCA, Spectroscopy

\section{Introduction}

Harriet Backer (1845-1932)

Harriet Backer is one of the most prominent painters of her generation in Norway $[1,2]$. Her artistic programme has been summarized as: "A classical, perspective composition combined with a modern apprehension of motif and colouring" [1]. In addition, Backer almost always painted on location, a common contemporary practice. A possible exception might have been The Altar at Uvdal Stave Church II (1910), which was finished in Backer's studio in Christiania (today, Oslo).

\section{Backer's paint box with colour tubes in Uvdal}

Between 1904 and 1909, Backer spent every summer in the medieval stave church at Uvdal in the Numedal valley in eastern Norway [2]. Since 1901,

\footnotetext{
*Correspondence: sara.mantellato@ifb.baug.ethz.ch

${ }^{2}$ Institut für Baustoffe (IfB), Physical Chemistry of Building Materials (PCBM), ETH Zürich, Stefano-Franscini-Platz 3, HIF B64.2, 8093 Zurich, Switzerland

Full list of author information is available at the end of the article
}

Fortidsminneforeningen (The National Trust of Norway) has owned the church [3]. This meant that the artist could turn the church into a studio without interruptions by church services. After her last stay in Uvdal and for more than 100 years, the artist's easel, stool and wooden box have been kept on site. The box contains 259 colour tubes, 20 bottles of Lefranc's Vernis à Peindre and a few paint rags [4] (Fig. 1). To our knowledge, this is the first study of this kind on this material.

The Uvdal box might have been Backer's regular container for all her church-related works. It is also likely that the artist used some or all of the Uvdal tubes for all paintings produced at Uvdal, and other church motifs.

During summers at Uvdal church, Backer produced one painting of its exterior that includes the churchyard and three colourful pictures of its Baroque interior (Fig. 2). Between 1891 and 1893 she made nine church paintings, within and outside the medieval church at Tanum (close to Christiania). She also painted six works between 1899 and 1903 in the medieval church at Stange (near Hamar and Lillehammer), and, in the period 1904-1908, 


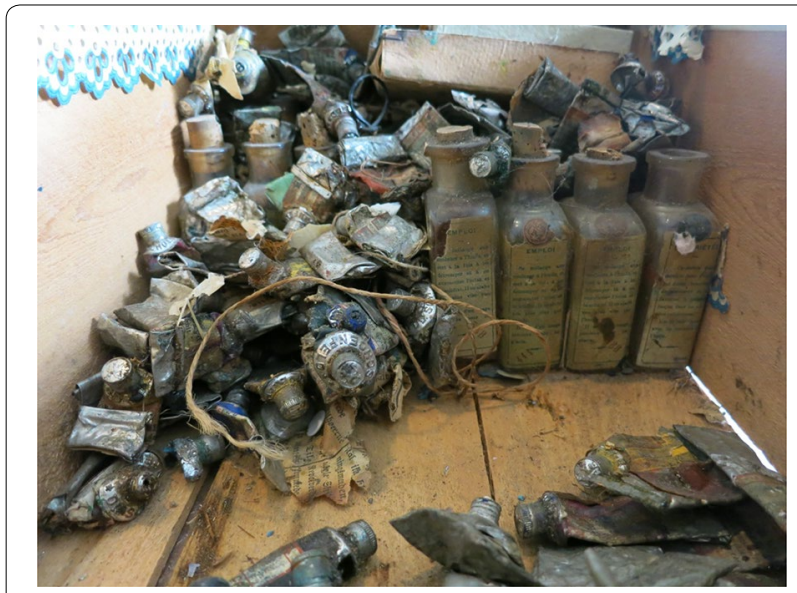

Fig. 1 Bottles of varnish discovered under the paint tubes at the Uvdal stave church. Photo courtesy of Merete Winness from Fortidsminneforeningen (The National Trust of Norway)

painted one in the Neo-Gothic Trinity church in Oslo [1]. In 1913, after her stay in Uvdal, Backer only painted one more church interior in the medieval St Mary's Church in Bergen.

Close examinations of the paint tubes showed that the majority were produced by the German manufacturer, Dr. Schoenfeld \& Co., Düsseldorf, currently known as Lukas-Nerchau (Fig. 3). To a lesser extent, the box also contains tubes from other German producers like C. Kreul Forchheim, C. Schmidt and H. Schmincke, in addition to the Belgian supplier J. Blockx and the French company Lefranc.

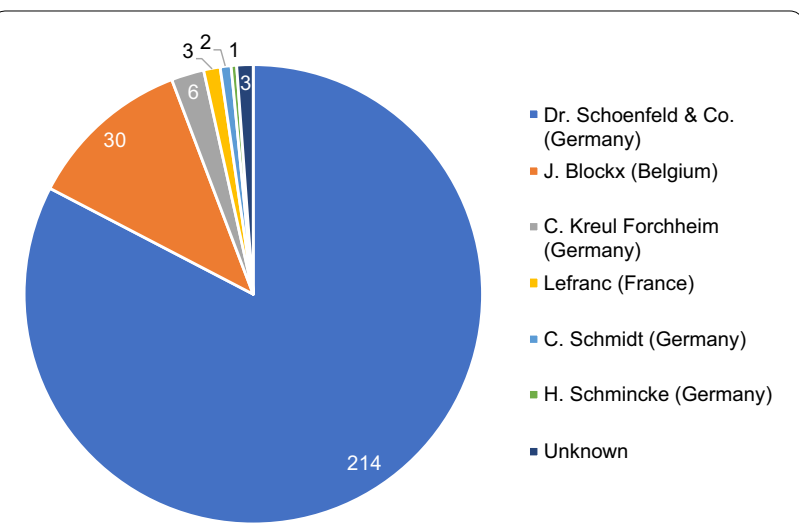

Fig. 3 Origin of Harriet Backer's paint tubes in the wooden box. The numbers in the pie chart indicate the number of tubes

\section{Aim of the study}

As will be shown in the next section reviewing the technical literature, many published works had the relatively limited-albeit relevant-aim to characterise the paints, without offering other insights into production aspects, analytical method development, degradation patterns and so on. In addition, no previous study sheds light on the quantification of the inorganic fraction of paint tubes. In view of this gap in knowledge, a validated ICP-OES method that can be applied on micro-samples of paint, and provides quantitative information on 17 elements, is presented here for the first time. Thanks to this method, this study focussed on the elemental characterisation (supported by multivariate analysis) of micro-samples from 57 tubes originally owned by Harriet Backer. The present investigation also represents the first systematic
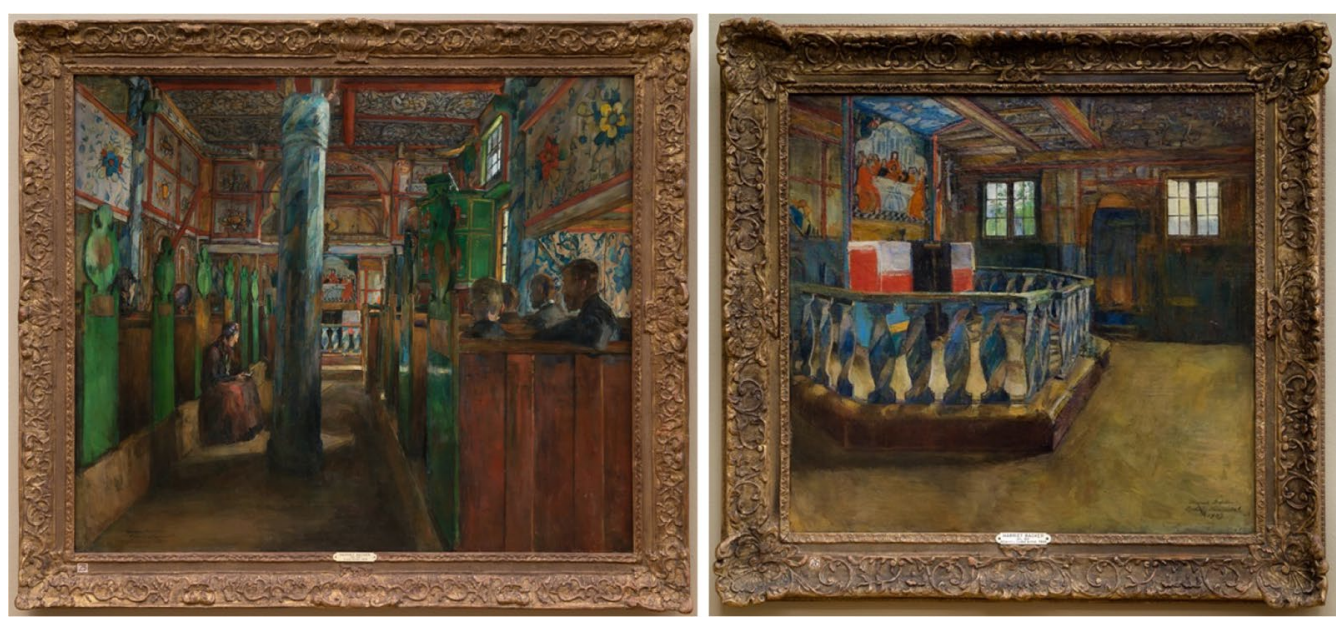

Fig. 2 Interior from Uvdal Stave Church (oil on canvas, $115 \times 135 \mathrm{~cm}^{2}$, OKODE, Bergen 2016) (left) and The Altar from Uvdal Stave Church I (oil on canvas, $80.5 \times 86 \mathrm{~cm}^{2}$, OKODE, Bergen 2016) (right) 
study on historical paint tubes by Dr. Schoenfeld. In addition, the comprehensive illustrated catalogue of the paint tubes owned by this Norwegian artist (attached to this paper as Additional file 1) complements the analytical results.

\section{Previous technical studies on oil paint tubes}

With the development of sophisticated, non- and microdestructive techniques in the last 30 years, there has been a significant increase in the number of studies on paint materials originally owned and used by artists. However, probably due to the limited availability of these materials, such studies are not numerous. As will be shown here, most have been mainly qualitative and focussed on the content of metal paint tubes. In fact, before the latter was invented in 1841 by John G. Rand [5-8], artists prepared or purchased paints that were mixed in small quantities, and slightly larger quantities could be stored for short periods in pig bladders.

A rapid (yet comprehensive) review of the literature on paint colour tubes, trying to highlight the aims of each article and to point out the role of the employed scientific methods, is here presented in chronological order. Townsend et al. studied late 19th century paint sample books by Winsor and Newton, and by Roberson, with the purposes of investigating differences between oil and watercolours, colour names referring to more than one pigment, adulterations and developments in manufacture. The work was conducted by a combination of visual, microscopic (stereo and polarized light microscopy (PLM)), spectroscopic (Fourier Transform Infrared (FTIR) spectroscopy, and energy dispersive X-ray (EDX) analysis) and chromatographic (thin layer chromatography) techniques [9].

Van De Laar and Burnstock examined pigments and binding media by the Dutch company, Claus and Fritz, from its 1921 line by scanning electron microscopy (SEM) coupled with EDX [10]. The Dutch artist, Georg Hendrik Breitner (1857-1923), mostly had Claus and Fritz's tubes in his paint box. In the notes on his sketchbook, Breitner mentioned H.C. Standage's "Artist manual of pigments". Similarly, Diependaal et al. carried out a multi-analytical study (PLM, X-Ray Fluorescence (XRF) spectroscopy, SEM-EDX, FTIR, Gas Chromatography coupled with Mass Spectrometry (GC-MS) and High Performance Liquid Chromatography (HPLC)) on Breitner's paint tubes (dating from 1897 to 1923), with reference to Standage's manual [11]. In the same work, one of the sampled tubes from Breitner's paint box was from Dr. Schoenfeld \& Co. Izzo and co-workers examined some Dutch case studies of modern oil paint tubes (dating 1920-1960) and paintings by XRF, SEM-EDX, attenuated total reflectance (ATR)-FTIR and GC-MS with the aim of elucidating their degradation phenomena [12]. With the latter technique, Pitthard and colleagues refined a method with a minimum of preliminary manipulation steps for the characterisation of the media of the oil paints used by Arnold Schönberg (1874-1951), and produced by the German company, Behrendt, at the beginning of the 20th century [13].

Cruz et al. used XRF to characterise some of the paints (by Winsor and Newton, Morin et Janet, C. Bourgès and Lefranc) used by Columbano Bordalo Pinheiro (1857-1929) around 1920 [14]. A more extensive study on paint materials containing ochres and earths used in Portugal at the end of the 19th century and beginning of 20th century was undertaken by Montagner et al. with micro-FTIR, Raman, micro-EDXRF and X-ray diffraction (XRD) [15]. With similar aims, FitzHugh et al. employed a multi-analytical approach (PLM, XRD, Raman spectroscopy and HPLC) to characterise the pigments in a paint box used by the American painter James McNeill Whistler (1834-1903), which now belongs to the Library of Congress in Washington, DC [16].

Wachowiak coupled Raman and XRF to study the preserved paint materials previously owned by the Polish painter, Jan Matejko (1838-1893), and produced by the French company, Richard Ainè [17]. A subsequent study on the same Matejko's paint tubes (in use from 1875 to 1890) by Otłowska et al. demonstrated the development of a liquid chromatography coupled with electrospray ionisation-mass spectrometry (LC-ESI-MS) method for the characterisation of the Indian Yellow dyestuff and its degradation products [18].

In the framework of a large European project on the spectroscopic study of the degradation process of chromium-based yellows in paintings by Vincent Van Gogh (1853-1890) [19-25], Monico et al. and Janssens et al. examined five historical paint tube samples. Two samples were provided by the Musée d'Orsay and allegedly coming from a paint tube used by Van Gogh himself (ca. 1890) and three others derived from other contemporary paint tubes. Their work relied on XRD, Raman, FTIR, Synchrotron Radiation-based (SR)-micro-XRD and SR-micro-X-Ray Absorption Near Edge Spectroscopy (XANES) to conclude that Van Gogh had different kinds of chrome yellow on his palette [21,25].

Otero et al. has recently published a vast and comprehensive study on the lead chromate pigments produced by Winsor and Newton [26, 27]. The authors characterised the pigments by a number of conventional and synchrotron radiation-based techniques (FTIR, Raman, UV-Vis reflectance, SR-micro-FTIR, SR-micro-XANES and SRmicro-XRF). With their results, Otero and co-workers were able to reconstruct the manufacturing processes of the pigments, as well as their degradation pathways. 
In 2009, conservators and researchers at the Munch Museum in Oslo, in collaboration with the Museum of Cultural History of the University of Oslo and the University of Pisa launched a project on paint tubes and working materials owned by Edvard Munch (18631944) [28-31]. With SEM-EDX, XRD, FTIR, Raman, GC- and HPLC-MS they studied the main components, admixtures, by-products and possible impurities in the tubes [29-31].

In the same year, Klempan and co-workers had the opportunity to study two paint boxes that belonged to the Canadian artist A.Y. Jackson (1882-1974) by a multi-analytical approach: SEM-EDX, XRD, FTIR, PLM and GC-MS. These paint boxes represented two different moments in Jackson's life and oeuvre: the period from 1936 to 1950 and from 1955 until 1968, when he was living and working in the Ottawa area. The extensive analytical characterization of the oil paints provided valuable information about Jackson's choices of materials in his artworks [32].

Even more recently, Christiansen et al. studied paint tubes and two paintings by P.S. Krøyer (1851-1909), which lent insight into the materials chosen by this Danish artist in the last part of his career [33]. This work relied on micro-Raman, SEM-EDX and HPLC, and in a subsequent study that focussed on the pigment in the tube labelled as "Jaune de Cadmium Citron" [34], they found that this contained a rare cadmium chromate compound $\left(\mathrm{KCd}_{2}\left(\mathrm{CrO}_{4}\right)_{2}\left(\mathrm{H}_{3} \mathrm{O}_{2}\right)\right)$. Thereafter they followed up with extensive characterisation of this pigment by micro-Raman, ATRFTIR, XRD and Electron Paramagnetic Resonance spectroscopy.

Rogge et al. recently characterised the oil paints produced by the American company Bocour between the 1940s and 80s [35]. Using FTIR, Raman and XRF the authors found several inconsistencies between the labels and the actual composition of the materials. Among other things, the authors suggested that such inconsistencies might constitute an issue in terms of preventive conservation.

Finally, Lee et al. recently characterised a collection of oil swatches by Winsor and Newton (dated between 1945 and 2003) with ESI-MS, FTIR, Thermogravimetric Analysis, GC-MS and HPLC-MS to infer information about the water sensitivity of modern oil paints $[36,37]$.

In summary, a large variety of techniques, both advanced and less advanced, has been used to characterise historical paint materials, with a special focus on the organic fraction. However, none of them has been employed to obtain accurate quantitative information nor information about Dr. Schoenfeld's historical oil paint tubes.

\section{Materials and methods}

\section{Sampling of the tubes}

Samples from 57 tubes (22\% of the grand total in the wooden box), one sample from each tube and each having a mass of $4 \mathrm{mg} \pm 3 \mathrm{mg}$, were taken under a Leica MZ6 stereomicroscope (Ortomedic AS, Lysaker, Norway) with the help of stainless-steel scalpels and dissecting needles. These were stored in snap cap glass vials. Samples were taken only from those tubes where the cap could be easily opened or those without cap, as indicated in Table 1.

Table 1 reports the colour of the sampled tubes (according to the order proposed in the conservation literature [38]), the producer, the number of the catalogue (see Additional file 1 for the complete one), the transcripts of the paper label, of the punching on collar and cap top, and the condition.

\section{Dissolution of the samples}

A protocol was developed and adopted for the dissolution of the samples prior to the ICP-OES analysis. Such an experimental procedure has been partly inspired by the ISO standard on the determination of major elements in solid biofuels [39], biofuels having a relatively similar matrix. It has to be noted that the quantity of the paint tube samples is two orders of magnitude lower than the one needed by the just mentioned ISO standard, adding, therefore, value to the analytical procedure that we developed for this work.

At first, the samples were weighed in clean, previously incinerated (at least $2 \mathrm{~h}$ at $550{ }^{\circ} \mathrm{C}$ in a muffle oven (B180, Nabertherm GmbH, Lilienthal, Germany)), and dry ceramic crucibles. The latter were then in the muffle oven for at least $5 \mathrm{~h}$ at $550{ }^{\circ} \mathrm{C}$. After the first incineration, the resulting ashes were moved with a magnesia stick. The crucibles with the samples were put in the muffle oven for a second incineration lasting at least $30 \mathrm{~min}$ at $550{ }^{\circ} \mathrm{C}$. After the second incineration, the samples were taken out of the oven and put in a desiccator with silica gel to cool down to room temperature. Afterwards, the ashes in the crucibles were weighed and treated with aqua regia (3 volumes of concentrated hydrochloric acid and 1 volume of concentrated nitric acid). ICP-grade nitric (TraceSELECT, $\geq 69 \%$ ) and hydrochloric (TraceSELECT, fuming, $\geq 37 \%$ ) acids were purchased from Fluka (Sigma-Aldrich Chemie GmbH, Buchs, Switzerland) for the preparation of the aqua regia. The ashes were left to react with aqua regia for several hours. The dispersions of aqua regia and ashes were subsequently weighed and filtered in clean PP containers with new sterile plastic syringes connected to $0.45 \mu \mathrm{m}$ CHROMAFIL Xtra H-PTFE-45/35 disposable syringe filters by Macherey-Nagel (MachereyNagel AG, Oensingen, Switzerland). A portion of the 
Table 1 Harriet Backer's paint tubes in Uvdal stave church: 57 of 259 were sampled for ICP-OES analyses

\begin{tabular}{|c|c|c|c|c|c|}
\hline Colour & Producer & No. & Paper label & Punching on collar \& cap top & Condition \\
\hline \multicolumn{6}{|l|}{ Violet } \\
\hline V & Schoenfeld & 1 & $\begin{array}{l}\text {....... Künstler ........ (in orange) } \\
\text { Violet oxide of c...ait (black) } \\
\text { Violette.. Koba...... (bold black) } \\
\text { Oxide violet de cobalt } \\
\text {.... di cobalto ...letto } \\
\text { Oxido de cobalto ....leta } \\
\text {.... Schoenfeld.......... (in orange) }\end{array}$ & $\begin{array}{l}\text { Letters on collar (Dr. Schoenfeld \& Co.) } \\
\text { plus one crowned lion holding an } \\
\text { anchor on cap top }\end{array}$ & $\begin{array}{l}\text { Tube end folded once, cap can be } \\
\text { opened }\end{array}$ \\
\hline V & Schoenfeld & 6 & $\begin{array}{l}\text {.... Künstler ........ } \\
\text { Violet Ultramari... } \\
\ldots \text {. letter Ultramar.... } \\
\text { Outremer vio... } \\
\text { Oltremare }{ }^{\prime} \ldots . \\
\text { Ultrama..... } \\
\ldots \ldots \ldots \ldots \ldots \ldots \ldots \ldots \ldots \ldots\end{array}$ & Letters & Tube end folded several times, no cap \\
\hline \multicolumn{6}{|l|}{ Blue } \\
\hline B & Schoenfeld & 1 & $\begin{array}{l}\ldots \text { Künstler Oelf ... (in orange) } \\
\text { Indigo (in black, bold) } \\
\ldots \text {. Düsseldorf (in orange) }\end{array}$ & $\begin{array}{l}\text { Letters on collar (Dr. Schoenfeld \& Co.) } \\
\text { plus one crowned lion holding an } \\
\text { anchor on cap top }\end{array}$ & $\begin{array}{l}\text { Tube end not folded, tight cap, big holes } \\
\text { in tube end }\end{array}$ \\
\hline B & Schoenfeld & 7 & $\begin{array}{l}\text {... Künstler Oelfa .... } \\
\text { Deep Ultramarine } \\
\text { Dunkler Ultramar.. } \\
\text { Outremer foncé } \\
\text { Oltremare scuro } \\
\text { Ultramar oscuro } \\
\ldots\end{array}$ & Letters \& lion & Tube end folded twice, no cap \\
\hline B & Schoenfeld & 8 & $\begin{array}{l}\text {... Künstler Oel ... } \\
\text { Deep Ultramarine } \\
\ldots \text {...ler Ultramar... } \\
\text { Outre ... } \\
\text { Oltr.. } \\
\text { Ultram .... } \\
\ldots\end{array}$ & Letters & Tube end folded once, no cap \\
\hline B & Schoenfeld & 13 & $\begin{array}{l}\text {...Künslrer Oel... } \\
\text { Deep Ultramarine } \\
\text {..nkler Ultramar... } \\
\text { Outremer fonc.. } \\
\text { Oltremare scuro } \\
\text { Ultramar oscuro } \\
\text {... Schoenfeld \& Co. ... }\end{array}$ & Letters & Tube end folded twice, no cap \\
\hline B & Schoenfeld & 16 & $\begin{array}{l}\text {... Oelf... } \\
\text { Deep Ultramarine } \\
\text {.kler Ultrama... } \\
\text { Outreemer foncé } \\
\text { Oltremare scuro } \\
\text { Ultramar oscur... } \\
\ldots \text {... Schoenfeld \& Co. Düss... }\end{array}$ & Letters & Tube end folded twice, no cap \\
\hline B & Schoenfeld & 25 & $\begin{array}{l}\ldots \ldots \ldots \text {.... (orange text?) } \\
\text { Deep Ultra... } \\
\text {...er Ultramar.. } \\
\text { Outremer foncé } \\
\text { Oltremare scuro } \\
\text { Ultramar oscuro } \\
\ldots \text {.. Schoenfeld \& Co. Düss... }\end{array}$ & Letters & Tube end folded once, no cap \\
\hline B & Schoenfeld & 42 & $\begin{array}{l}. . e \text { eineste Künstler Oelfar... } \\
\text { Cobalt Blue light } \\
\text { Kobaltblau } 2 \text { hell } \\
\text { Bleu de Cobalt clair } \\
\text { Bleu cobalto chiaro } \\
\text { Azul de cobalto clar.. } \\
\text {... Schoenfeld \& Co. Dü....... }\end{array}$ & Letters & Tube end not folded, no cap \\
\hline
\end{tabular}


Table 1 (continued)

\begin{tabular}{|c|c|c|c|c|c|}
\hline Colour & Producer & No. & Paper label & Punching on collar \& cap top & Condition \\
\hline B & Schoenfeld & 43 & $\begin{array}{l}\text {.obalt Blue light } \\
\text {..baltblau } 2 \ldots \\
\text { Bleu de Cobalt c.... } \\
\text { Bleu cobalto chi.... } \\
. . z u l \text { de cobalto cla... } \\
\ldots . . . . \text { feld \& Co. Dü....... }\end{array}$ & Letters & Tube end folded twice, no cap \\
\hline B & Schoenfeld & 51 & $\begin{array}{l}\text { Brilliant Bl............ (orange) } \\
\ldots \text {.illant b..... } \\
\text { Dr. Schoenfeld ........ (orange) } \\
\text { Düsseldorf (orange) }\end{array}$ & Letters & Tube end folded once, no cap \\
\hline B & Schoenfeld & 53 & $\begin{array}{l}\text {....................... (orange) } \\
\text {.rillant blue } \\
\text { Bleu brilliant.... } \\
\ldots \text {.. Schoenf........... (orange) } \\
\text { Düsseldorf (orange) }\end{array}$ & Letters & Tube end folded twice, no cap \\
\hline B & Schoenfeld? & 55 & 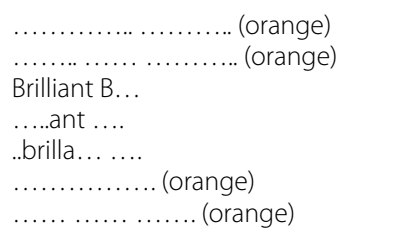 & No collar, no cap & No collar, no cap \\
\hline B & Schoenfeld & 56 & $\begin{array}{l}\ldots \ldots \ldots \ldots \ldots \ldots \text { (orange) } \\
\ldots \ldots \ldots \text { Blue } \\
\ldots \text { ant } b \ldots \ldots \\
\ldots \ldots \ldots \ldots \ldots \ldots \\
\ldots \ldots \ldots \ldots \ldots \ldots \text { (orange) } \\
\ldots \ldots \ldots \ldots \ldots \ldots \text { (orange) }\end{array}$ & Letters \& lion & $\begin{array}{l}\text { Cap fell off during handling, end of tube } \\
\text { is missing }\end{array}$ \\
\hline B & Schoenfeld & 57 & No label & Letters & Tube end folded several times, no cap \\
\hline B & Schoenfeld & 64 & No label & ? \& lion & $\begin{array}{l}\text { Tube end folded twice, blue paint in } \\
\text { one hole }\end{array}$ \\
\hline \multicolumn{6}{|l|}{ Green } \\
\hline G & Schoenfeld & 1 & $\begin{array}{l}\text { Feinste Künstler Oelf...... (orange) } \\
\text { Terre Verte (black) } \\
\text { Grüne Erde } \\
\text { Terre verte naturelle } \\
\text { Terra verde } \\
\text { Thierra verde } \\
\text {.... Schoenfeld \& C.. (Orange) } \\
\text { Düsseldorf (orange) }\end{array}$ & Letters & Tube end folded once, no cap \\
\hline G & Schoenfeld & 2 & $\begin{array}{l}\text { Terre Verte (black) } \\
\text { Grüne Erde } \\
\text { Terre verd... naturelle } \\
\ldots . . . \text { erde } \\
\text {...... erde } \\
\text {.... Schoenfeld ..... Düsseldorf }\end{array}$ & Letters \& lion & $\begin{array}{l}\text { Tube end folded several times, cap can } \\
\text { be opened }\end{array}$ \\
\hline G & Schoenfeld & 6 & $\begin{array}{l}\text {....... Künstler ....... } \\
\text { Terre Verte } \\
\text { Grüne E.... } \\
\text { Terre verte naturel.. } \\
\text { Terra verde } \\
\text { Thierra verde } \\
\ldots \ldots \ldots . . . \text { enfeld \& Co. .......... }\end{array}$ & Letters \& lion & $\begin{array}{l}\text { Tube end folded twice, cap can be } \\
\text { opened }\end{array}$ \\
\hline
\end{tabular}


Table 1 (continued)

\begin{tabular}{|c|c|c|c|c|c|}
\hline Colour & Producer & No. & Paper label & Punching on collar \& cap top & Condition \\
\hline G & Schoenfeld & 7 & $\begin{array}{l}\ldots \ldots \ldots \ldots \ldots \ldots \\
. . e r r e ~ v e r . . . . \\
. . r u ̈ n e \\
\text { Terre verte na........ (orange) } \\
\text { Terra verde } \\
\text { Thierra ver.... } \\
\ldots \ldots \ldots \ldots \text {............. }\end{array}$ & Letters \& lion & $\begin{array}{l}\text { Tube end folded several times, cap can } \\
\text { be opened }\end{array}$ \\
\hline G & Schoenfeld & 9 & $\begin{array}{l}\ldots \ldots \ldots \ldots . \ldots \ldots \text { (orange) } \\
\ldots . . \text { Künstl......... (orange) } \\
\text { Viridian } \\
\text { Chromoxydgrün } \\
\text { Vert.. emera........ } \\
\text { Verde smeral...... } \\
\text { Verde esmer..... } \\
\ldots \text { Schoenfeld \& Co. ......... }\end{array}$ & Letters \& lion & $\begin{array}{l}\text { Tube end folded twice, cap can be } \\
\text { opened }\end{array}$ \\
\hline G & Schoenfeld & 10 & 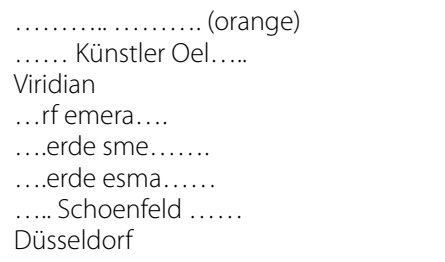 & Letters \& lion & $\begin{array}{l}\text { Tube end folded once, cap can be } \\
\text { opened }\end{array}$ \\
\hline G & Schoenfeld & 12 & $\begin{array}{l}\ldots \ldots \ldots \ldots \ldots \ldots . \text { (orange) } \\
\ldots \ldots \ldots \ldots \ldots \ldots \text { (orange) } \\
\text { Viridian } \\
\ldots \ldots \text { moxydgrün .... } \\
\ldots \ldots \ldots \ldots \ldots \\
\ldots \ldots \text { verd.. esmera..... } \\
\ldots \ldots \ldots \ldots \ldots \ldots \ldots \ldots \text { (orange) }\end{array}$ & Letters \& lion & $\begin{array}{l}\text { Tube end folded twice, cap can be } \\
\text { opened }\end{array}$ \\
\hline G & Schoenfeld & 15 & $\begin{array}{l}\text { No label - green paint around collar } \\
\text { tip }\end{array}$ & Letters \& lion & $\begin{array}{l}\text { Tube end folded once, cap can be } \\
\text { opened }\end{array}$ \\
\hline \multicolumn{6}{|l|}{ Yellow } \\
\hline Y & Schoenfeld & 8 & $\begin{array}{l}\ldots \ldots \ldots \ldots \text { (orange) } \\
\ldots \ldots \ldots \ldots \ldots \text { (orange) } \\
\ldots . . \text { iante Yellow deep (black) } \\
\ldots \ldots \ldots \text { dun..... } \\
\ldots . \text { me brillan....... } \\
\ldots . \text { Brillante scuro } \\
\ldots \ldots \text { brillante os..... } \\
\ldots \ldots \ldots \ldots \ldots \ldots \ldots \ldots \text { (orange) }\end{array}$ & Letters & Tube end folded twice, no cap \\
\hline Y & Schoenfeld & 9 & 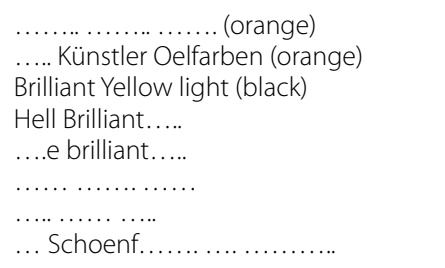 & Letters & Tube end folded twice, no cap \\
\hline Y & C. Schmidt & 27 & $\begin{array}{l}\text {..ar... Fabrik (black) } \\
\text {......mgelb hell } \\
\text { Ja.... Chrôme } \\
\text {...t, Düssel.... (black) }\end{array}$ & $\begin{array}{l}\text { No letters on collar, no punching on } \\
\text { cap top }\end{array}$ & Tube end not folded, cap can be opened \\
\hline Y & Schoenfeld & 28 & Yellow label with no text & Letters \& lion & $\begin{array}{l}\text { Tube end folded several times, cap can } \\
\text { be opened }\end{array}$ \\
\hline Y & Schoenfeld & 30 & $\begin{array}{l}\text { Label lost and yellow paint all over the } \\
\text { Tube end }\end{array}$ & Letters & Tube end folded once, no cap \\
\hline Y & Schoenfeld & 44 & No label & Schoenfeld across lower end of tube & $\begin{array}{l}\text { Tube end slightly folded, no cap, yellow } \\
\text { paint on top of tube end }\end{array}$ \\
\hline
\end{tabular}


Table 1 (continued)

\begin{tabular}{|c|c|c|c|c|c|}
\hline Colour & Producer & No. & Paper label & Punching on collar \& cap top & Condition \\
\hline Y & C. Schmidt & 46 & $\begin{array}{l}\text { Farben Fabrik } \\
\text { Lichter Ocker } \\
\text { Ochre claire } \\
\text { C. Schmidt, Düsseldorf }\end{array}$ & No punching & $\begin{array}{l}\text { Tube end folded twice, cap can be } \\
\text { opened }\end{array}$ \\
\hline \multicolumn{6}{|c|}{ Yellowish Red } \\
\hline$Y-R$ & Schoenfeld & 3 & $\begin{array}{l}\text {........ Künstler Oelfar.... } \\
\text { Naples Yellow reddish } \\
\text { Rötlich Neapelgelb } \\
\text { Jaune de Naples roug.átre } \\
\text { Giallo di Napoli ross..... } \\
\text { Amanillo de Napoles ...... } \\
\text { Dr... Schoenfeld \& Co. Düsseldorf }\end{array}$ & Letters & Tube end slightly folded, no cap \\
\hline Y-R & Schoenfeld & 6 & 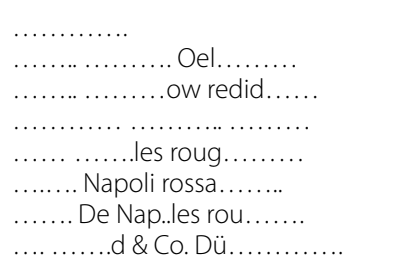 & Letters & Tube end folded twice, no cap \\
\hline $\mathrm{Y}-\mathrm{R}$ & Schoenfeld & 10 & 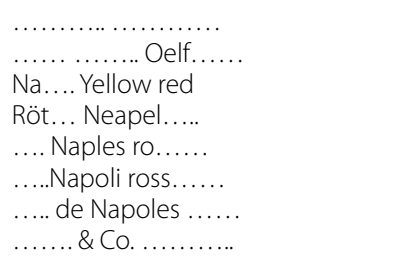 & Letters & Tube end folded twice, no cap \\
\hline$Y-R$ & Schoenfeld & 11 & $\begin{array}{l}\text { Nöles Yellow reddish } \\
\text { Rötclich Neaplegelb } \\
\text { Jeune de Naples rou....... } \\
\ldots \ldots \ldots . \text { di Napoli rossastro } \\
\text { Amanillo de Napoles rofizo } \\
\text {.... Schoenfeld \& Co....... }\end{array}$ & Letters & Tube end folded twice, no cap \\
\hline \multicolumn{6}{|l|}{ Red } \\
\hline $\mathrm{R}$ & Schoenfeld & 3 & $\begin{array}{l}\text { Feinste Künstler Oelfarben } \\
\text { Pink Madder } \\
\text { Krapplack } 4 \text { hel..I } \\
\text { Laque de garance rose ..... } \\
\text { Lacca di garance chiaro rosa } \\
\text { Laca de garanza rosa dora } \\
\text {.... Schoenfeld \& Co. D........ }\end{array}$ & Letters \& lion & $\begin{array}{l}\text { Tube end slightly folded, cap can be } \\
\text { opened }\end{array}$ \\
\hline $\mathrm{R}$ & Schoenfeld & 5 & $\begin{array}{l}\text {.... Künstler Oe...... } \\
\text { Pink Madder } \\
\text { Krapplack } 4 \text { heller } \\
\text { Laque de garance rose ....ore } \\
\text { Lacca.. garance chiara ros..dorata } \\
\text { Laca de garanze rosa ... rada } \\
\text {..... Schoenfeld \& Co. Düss........ }\end{array}$ & Letters & Tube end not folded, no cap \\
\hline R & Schoenfeld & 9 & 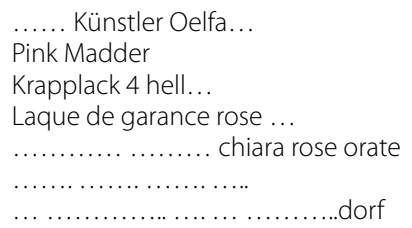 & Letters \& lion & $\begin{array}{l}\text { Tube end folded twice, cap can be } \\
\text { opened }\end{array}$ \\
\hline
\end{tabular}


Table 1 (continued)

\begin{tabular}{|c|c|c|c|c|c|}
\hline Colour & Producer & No. & Paper label & Punching on collar \& cap top & Condition \\
\hline $\mathrm{R}$ & Schoenfeld & 14 & $\begin{array}{l}\text { Pink Madder } \\
\text { Krapplack } 4 \text { heller } \\
\text { Laque de garance rose ....ré } \\
\text { Lacca di garance chiaro rose dorata } \\
\text { Laca de garanza rosa dorada } \\
\text { Dr... Schoenfeld \& Co. Düsseldorf }\end{array}$ & Letters \& lion & $\begin{array}{l}\text { Tube end slightly folded, cap can be } \\
\text { opened }\end{array}$ \\
\hline $\mathrm{R}$ & Schoenfeld & 15 & $\begin{array}{l}\text {...... Künstler .......... } \\
\text { Pink Madder } \\
\text { Krapplack } 4 \text { hell.. } \\
\text { Laque de garance rose d.... } \\
\text { Lacca.. garanco chiaro rosa } \\
\text { Laca.. garanza rosa ...... } \\
\text {.... Schoenfeld \& Co. Düsseldorf }\end{array}$ & Letters & Tube end slightly folded, no cap \\
\hline $\mathrm{R}$ & Schoenfeld & 16 & $\begin{array}{l}\text {................... (orange) } \\
\text { Pink Madder } \\
\text { Krapplack } 4 \text { hel... } \\
\text { Laque de garance rose ..... } \\
\text { Lacca di garance chiaro rosa } \\
\text { Laca de garanza rosa } \\
\text {...........enfeld \& Co. D...... dorf }\end{array}$ & Letters \& lion & $\begin{array}{l}\text { Tube end slightly folded, cap can be } \\
\text { opened }\end{array}$ \\
\hline $\mathrm{R}$ & Schoenfeld & 17 & $\begin{array}{l}\text { Pink Madder } \\
\text { Krapplack } 4 \text { hel..... } \\
\text { Laque de garance rose dorata } \\
\text { Lacca di garance chiaro rosa } \\
\text { Laca de garanze rosa } \\
\text { Dr... Schoenfeld ............... }\end{array}$ & Letters \& lion & $\begin{array}{l}\text { Tube end slightly folded, cap can be } \\
\text { opened }\end{array}$ \\
\hline $\mathrm{R}$ & Schoenfeld & 25 & 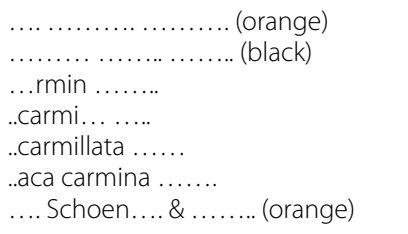 & Letters \& lion & $\begin{array}{l}\text { Tube end slightly folded, cap can be } \\
\text { opened }\end{array}$ \\
\hline $\mathrm{R}$ & Schoenfeld & 29 & $\begin{array}{l}\text {...................... (orange) } \\
\text { Karmin Lack ..... } \\
\text {.... Carmine sur....... } \\
\ldots . . \text { carminala sopra..... } \\
\text { Laca carminada ....... } \\
\text {.... Schoenfeld \& Co. Dü........ }\end{array}$ & Letters \& lion & $\begin{array}{l}\text { Tube end slightly folded, cap can be } \\
\text { opened }\end{array}$ \\
\hline $\mathrm{R}$ & Schoenfeld & 30 & $\begin{array}{l}\ldots \ldots . . \text { Künstler ......... } \\
\ldots \ldots \text { crimson ....... } \\
\ldots \text { armin.......... } \\
\ldots . \text { Carmine sur..... } \\
\ldots . \text { Carminara so......... } \\
\text { Laca carmina ....... } \\
\ldots . \text { Schoenfeld \&........ }\end{array}$ & Letters \& lion & $\begin{array}{l}\text { Tube end slightly folded, cap can be } \\
\text { opened }\end{array}$ \\
\hline $\mathrm{R}$ & Schoenfeld & 43 & Small fragment of red inner label & Letter \& lion & $\begin{array}{l}\text { Tube end folded several times, cap can } \\
\text { be opened, red paint on collar }\end{array}$ \\
\hline $\mathrm{R}$ & Schoenfeld & 45 & $\begin{array}{l}\text { Finest crimson lake } \\
\text { Karminlack } \\
\text { Laque carminée surfine } \\
\text { Lacca carminata sopraffina } \\
\text { Laca carminada } \\
\text { Dr.. Schoenfeld \& Co. Düsseldorf }\end{array}$ & Letters \& lion & $\begin{array}{l}\text { Tube end slightly folded, cap can be } \\
\text { opened }\end{array}$ \\
\hline
\end{tabular}


Table 1 (continued)

\begin{tabular}{|c|c|c|c|c|c|}
\hline Colour & Producer & No. & Paper label & Punching on collar \& cap top & Condition \\
\hline R & Schoenfeld & 46 & $\begin{array}{l}\text { Chinese Vermilion } \\
\text { Chinesischer Zinnober } \\
\text { Vermillion de Chine } \\
\text { Vermiglione di China } \\
\text {..ermellon chino } \\
\text {.. Schoenfeld \& Co. Düss... }\end{array}$ & Letters \& lion & $\begin{array}{l}\text { Tube end slightly folded, cap can be } \\
\text { opened }\end{array}$ \\
\hline \multicolumn{6}{|l|}{ Brown } \\
\hline Bro & Schoenfeld & 7 & $\begin{array}{l}\ldots \ldots . \ldots \ldots \ldots \\
\ldots \ldots . \text { Green Ea.... } \\
\ldots . \text { br. grüne ....... } \\
\text { Terre verte bru..... } \\
\text { Terra verde ...... } \\
\text {.ierra verd....... } \\
\ldots \ldots \ldots \ldots \ldots \ldots \ldots \ldots \ldots \ldots \ldots\end{array}$ & Letters \& lion & $\begin{array}{l}\text { Tube end folded several times, cap can } \\
\text { be opened }\end{array}$ \\
\hline Bro & Schoenfeld & 9 & $\begin{array}{l}\ldots \ldots \ldots \ldots \ldots \ldots \ldots \ldots \ldots \\
\ldots \ldots \ldots \ldots \text { Umber } \\
\ldots \ldots \ldots \ldots \ldots \ldots \ldots \ldots \\
\ldots \ldots \ldots \ldots \ldots \ldots \ldots \ldots\end{array}$ & Letters & $\begin{array}{l}\text { Tube end folded several times, no cap, } \\
\text { brown paint in opening }\end{array}$ \\
\hline Bro & Schoenfeld & 10 & No label & Letters & $\begin{array}{l}\text { Tube end folded several times, no cap, } \\
\text { brown paint in opening }\end{array}$ \\
\hline Bro & Schoenfeld & 11 & No label & Letters & $\begin{array}{l}\text { Tube end folded several times, no cap, } \\
\text { brown paint in opening }\end{array}$ \\
\hline Bro & Schoenfeld & 12 & $\begin{array}{l}\text { Only a tiny fragment of a brownish } \\
\text { inner label }\end{array}$ & Letters & $\begin{array}{l}\text { Tube end folded several times, no cap, } \\
\text { brown paint in opening }\end{array}$ \\
\hline Bro & Schoenfeld & 13 & No label & Letters \& lion & $\begin{array}{l}\text { Tube end not folded, cap can be opened, } \\
\text { brown paint on collar }\end{array}$ \\
\hline \multicolumn{6}{|l|}{ White } \\
\hline W & Blockx & 6 & $\begin{array}{l}\text {.... D'a......... } \\
\text {....kx, Fils, Vieux-Dieu (B...... }\end{array}$ & No letters on collar. & Tube end folded several times, no cap \\
\hline W & Blockx & 13 & $\begin{array}{l}\text {.... D'argent Moyen } \\
\text { J. Blockx Fils, Vieux-Dieu }\end{array}$ & No letters on collar & Tube end folded several times, no cap \\
\hline W & Blockx & 27 & $\begin{array}{l}\text {... ARGENT ... } \\
\text { (the rest of the label is covered by } \\
\text { paper or similar (the latter may hide } \\
\text { the rest of the label)) } \\
\text { This is the largest tube in Harriet } \\
\text { Backer's box }\end{array}$ & No letters on collar \& B on cap top & $\begin{array}{l}\text { Tube end folded several times, tight cap } \\
\text { (white paint on top and at the bottom) }\end{array}$ \\
\hline
\end{tabular}

This list reports the transcript of the paper labels and the punching on collars and on the top of the caps. The number indicates the number of the catalogue and the long ellipses the absence of any recognisable text

filtered solution was then diluted with $2 \%(\mathrm{w} / \mathrm{w}) \mathrm{HNO}_{3}$ in ultrapure water (UPW). In order for the concentration of the elements to be in the linear range of the ICP-OES method, the dilutions of the samples ranged from around 300 to 3300 times. The UPW $(\rho=18.2 \mathrm{M} \Omega \mathrm{cm}$, TOC around 1-2 ppb) was dispensed by a Milli-Q A+ water purification system from MilliPore (Merck \& Cie, Schaffhausen, Switzerland). Fresh $2 \%(\mathrm{w} / \mathrm{w}) \mathrm{HNO}_{3}$ in UPW was the blank used for the analyses.

\section{Instrumental conditions}

A Thermo Scientific iCAP 6300 Dual View ICP-OES (Thermo Fisher Scientific Inc., Waltham, MA, USA) with a CETAC ASX-260 autosampler (CETAC, Omaha, NE,
USA) was used for the analyses. The chosen operating conditions come from previous work on complex matrices [40]. These are reported in Table 2.

The Windows programs used for the ICP-OES analyses were iTEVA 2.8.0.97 and Qtegra 2.8.2944.202 (both from Thermo Fisher Scientific Inc.).

\section{Standard and reference solutions}

As shown in Table 3, a multi-elemental standard solution and reference solution were prepared by suitably diluting multielement standard solution 1 for ICP (TraceCERT, in $10 \%$ nitric acid, Fluka) and $1000 \mathrm{mg} / \mathrm{L}$ silicon, sulfur and phosphorus standard bulk solutions for ICP (TraceCERT, in $2 \%$ nitric acid, Fluka) in $2 \%(\mathrm{w} / \mathrm{w}) \mathrm{HNO}_{3}$ in UPW. 
Table 2 Values of operating conditions and types of instrument parts employed for the ICP-OES analyses in this work

\begin{tabular}{ll}
\hline $\begin{array}{l}\text { Operating condition/instrument } \\
\text { part }\end{array}$ & Value/type \\
\hline Effective focal length & $383 \mathrm{~nm}$ \\
Spectral range & $166-847 \mathrm{~nm}$ \\
Detector & CID86 chip (charge injection device) \\
RF generator & $27.12 \mathrm{MHz}$ solid state \\
Plasma viewing & Dual \\
Plasma and shear gas & Argon \\
Nebulizer & Burgener MiraMist High \\
& Solids Nebulizer (0.4-2.0 mL/min) \\
Spray chamber & Glass cyclone \\
Plasma torch & Enhanced matrix tolerance (EMT) \\
& semi-demountable \\
RF power & $1150 \mathrm{~W}$ \\
Pump rate & $50 \mathrm{rpm}$ \\
Auxiliary gas flow & $0.5 \mathrm{~L} /$ min \\
Nebulizing gas flow & $0.5 \mathrm{~L} /$ min \\
Number of replicates per sample & 3 \\
Maximum integration time for low & $15 \mathrm{~s}$ (both axial and radial) \\
WL range & \\
Maximum integration time for high & $10 \mathrm{~s}$ (axial) and $5 \mathrm{~s}$ (radial) \\
WL range & $45 \mathrm{~s}$ \\
Flush time &
\end{tabular}

Table 3 Composition of the 17-elements standard and reference solutions used in this study

\begin{tabular}{lcl}
\hline Element & Standard $(\boldsymbol{\mu g} / \mathbf{L})$ & Reference $(\boldsymbol{\mu g} / \mathbf{L})$ \\
\hline $\mathrm{Al}$ & 1000 & 500 \\
$\mathrm{Ba}$ & 200 & 100 \\
$\mathrm{Cd}$ & 200 & 100 \\
$\mathrm{Co}$ & 200 & 100 \\
$\mathrm{Cr}$ & 1000 & 500 \\
$\mathrm{Cu}$ & 200 & 100 \\
$\mathrm{Fe}$ & 200 & 100 \\
$\mathrm{~K}$ & 2000 & 1000 \\
$\mathrm{Mg}$ & 200 & 100 \\
$\mathrm{Mn}$ & 200 & 100 \\
$\mathrm{Na}$ & 1000 & 500 \\
$\mathrm{P}$ & 1001 & 500.5 \\
$\mathrm{~Pb}$ & 2000 & 1000 \\
$\mathrm{~S}$ & 1000 & 500 \\
$\mathrm{Si}$ & 1001 & 500.5 \\
$\mathrm{Sr}$ & 200 & 100 \\
$\mathrm{Zn}$ & 200 & 100
\end{tabular}

The blank is $2 \%(\mathrm{w} / \mathrm{w}) \mathrm{HNO}_{3}$ in UPW
Principal component analysis (PCA)

Principal component analysis (PCA) is a statistical technique that enables exploration and recognition of patterns in multivariate data whose variables show correlation. By carrying out PCA, noise and meaningless data variations are often easily discerned. In a nutshell, PCA solves a maximization problem. In practice, PCA computes a geometrical transformation to the variables so as to obtain new and uncorrelated variables (the socalled principal components, PCs) that express the maximum possible variance (namely, how the information is dispersed within the data [41]). The result of PCA is a socalled "score plot" that projects the data in the new space of the PCs. The score plot represents, with a few numbers, data that could not possibly be visualized because of their multidimensionality. Besides, it also identifies possible (and hidden) clusters and outliers [42-46].

PCA was carried out using the "prcomp" command of $\mathrm{R}$ 3.5.1 [47]. RStudio 1.1.383 was used as GUI [48]. As will be clear from the Results and Discussion section of the paper, the results for each element (above the limits of quantification, LOQs) are presented as normalised percentages. The matrix dataset for the PCA is composed by such normalised percentages for the 17 analysed elements. In the computing of the PCA, data were normalised and scaled.

\section{Results and discussion}

\section{Analytical figures of merit of the ICP-OES method}

Table 4 reports the selected spectral lines, their view mode, the percentage deviation between the measured and the expected concentrations of the above-mentioned reference solution (namely, a measurement of the trueness $[44,49]$ of the method). This is based on the optimization of the ICP-OES method (in terms of spectral lines, spectral and matrix interferences, linear response, and sensitivity). Also reported in the same table are the obtained limits of detection (LODs) and LOQs, corresponding to two quantities that define the concentrations at which an analyte can be detected and quantified with a given level of confidence, respectively $[50,51]$.

Table 5 reports the average coefficient of variation (CV) for each element and each paint colour. CV is a measurement of the precision [52] of the method and-along with the trueness-contributes to its accuracy [40, 49].

The ICP-OES method developed for this work can be considered highly accurate, with values of closeness of agreement never above $6.0 \%$ and $\mathrm{CV}$ of the measurements rarely exceeding $10 \%$, well below the $15 \%$ for both trueness and precision reported in the literature [53]. 
Table 4 Measures of closeness of agreement, LODs and LOQs obtained with the proposed method

\begin{tabular}{|c|c|c|c|c|c|}
\hline Element & Spectral line $(\mathrm{nm})$ & View mode & $\begin{array}{l}\text { Closeness of agreement } \\
\text { (\%) }\end{array}$ & LOD $(\mu \mathrm{g} / \mathrm{L})$ & $\mathrm{LOQ}(\mu \mathrm{g} / \mathrm{L})$ \\
\hline $\mathrm{Al}$ & 396.152 & Axial & 4.6 & 0.4 & 1.4 \\
\hline $\mathrm{Ba}$ & 455.403 & Axial & 4.5 & 0.03 & 0.10 \\
\hline $\mathrm{Cd}$ & 228.802 & Axial & 2.6 & 0.1 & 0.5 \\
\hline Co & 228.616 & Axial & 2.9 & 0.2 & 0.8 \\
\hline $\mathrm{Cr}$ & 283.563 & Axial & 4.0 & 0.2 & 0.8 \\
\hline $\mathrm{Cu}$ & 324.754 & Axial & 4.0 & 0.3 & 1.0 \\
\hline $\mathrm{Fe}$ & 259.940 & Axial & 1.1 & 0.2 & 0.7 \\
\hline K & 766.490 & Axial & 1.6 & 0.4 & 1.4 \\
\hline Mg & 279.553 & Radial & 2.7 & 0.09 & 0.31 \\
\hline $\mathrm{Mn}$ & 257.610 & Axial & 3.2 & 0.07 & 0.23 \\
\hline $\mathrm{Na}$ & 589.592 & Radial & 4.4 & 13 & 45 \\
\hline$P$ & 177.495 & Axial & 0.2 & 0.7 & 2.2 \\
\hline $\mathrm{Pb}$ & 220.353 & Axial & 2.9 & 1.1 & 3.6 \\
\hline S & 182.034 & Axial & 2.6 & 1.8 & 6.0 \\
\hline Si & 251.611 & Axial & 1.8 & 0.8 & 2.8 \\
\hline Sr & 407.771 & Axial & 3.3 & 0.03 & 0.11 \\
\hline $\mathrm{Zn}$ & 213.856 & Axial & 6.0 & 0.2 & 0.6 \\
\hline
\end{tabular}

The closeness of agreement was computed as the mean percentage deviation from the expected value for the mean of 11 independently prepared reference solutions. For the LOD and LOQ, $n_{\text {blanks }}=10$. For the calculations of the closeness of agreement, LOD and LOQ, deviant values were discarded according to the ISOrecommended Grubbs' test with $P=0.05$ [44]

Our method can also be considered very sensitive, being the obtained LODs in line or below (excluding those for magnesium, potassium, sodium and strontium) the general LODs of the elements using the most sensitive lines in ICP-OES $[54,55]$. In the case of phosphorus, our
LOD is better than the general one of a factor close to 6 . In addition, the 11 measurements of closeness of agreement were taken during different sessions in different days using newly prepared reference solutions, still giving satisfactory results on average and proving that it is

Table 5 Average CV obtained on the determinations for each element and each paint colour

\begin{tabular}{|c|c|c|c|c|c|c|c|}
\hline Element & Violet & Blue & Green & Yellow & Yellowish-Red & Brown & White \\
\hline $\mathrm{Al}$ & $0.5 \% *$ & $0.9 \%$ & $2.6 \%$ & $2.5 \%$ & $4.9 \%$ & $0.8 \%$ & $3.6 \%$ \\
\hline $\mathrm{Ba}$ & $2.2 \% *$ & $1.2 \%$ & $1.5 \%$ & $1.2 \%$ & $8.0 \%$ & $0.4 \%$ & $0.6 \%$ \\
\hline $\mathrm{Cd}$ & $0.4 \% *$ & $5.2 \%$ & $4.6 \%$ & $2.6 \%$ & $1.9 \% *$ & $3.8 \%$ & $15.4 \% *$ \\
\hline Co & $0.3 \%^{*}$ & $2.5 \%$ & - & - & - & - & $8.0 \% *$ \\
\hline $\mathrm{Cr}$ & $25.9 \% *$ & $6.0 \%$ & $3.0 \%$ & $4.5 \%$ & $2.7 \%$ & $2.8 \%$ & $4.6 \% *$ \\
\hline $\mathrm{Cu}$ & $3.7 \% *$ & $2.7 \%$ & $2.3 \%$ & $7.4 \%$ & $2.3 \% *$ & $2.3 \%$ & $5.8 \%$ \\
\hline $\mathrm{Fe}$ & $4.6 \% *$ & $1.3 \%$ & $1.8 \%$ & $1.5 \%$ & $4.9 \%$ & $0.4 \%$ & $2.0 \%$ \\
\hline K & $17.9 \% *$ & $1.7 \%$ & $1.9 \%$ & $4.1 \%$ & $7.1 \%$ & $0.8 \%$ & $8.3 \%$ \\
\hline $\mathrm{Mg}$ & $1.2 \% *$ & $1.5 \%$ & $1.8 \%$ & $2.2 \%$ & $3.1 \%$ & $1.3 \%$ & $10.6 \%$ \\
\hline $\mathrm{Mn}$ & $0.6 \% *$ & $3.5 \%$ & $0.5 \%$ & $3.9 \%$ & - & $0.7 \%$ & - \\
\hline $\mathrm{Na}$ & $1.3 \% *$ & $3.9 \%$ & $6.4 \%$ & $6.5 \%$ & $4.0 \%$ & $10.8 \%$ & $12.1 \% *$ \\
\hline$P$ & $0.6 \% *$ & $7.7 \%$ & $8.7 \%$ & $7.9 \%$ & - & $2.5 \%$ & $2.0 \% *$ \\
\hline $\mathrm{Pb}$ & $5.0 \% *$ & $3.6 \%$ & $3.4 \%$ & $1.6 \%$ & $0.4 \%$ & $3.8 \%$ & $0.2 \%$ \\
\hline S & $2.7 \% *$ & $4.8 \%$ & $6.2 \%$ & $4.1 \%$ & $4.2 \%$ & $0.8 \%$ & $6.7 \%$ \\
\hline $\mathrm{Si}$ & $2.5 \% *$ & $2.2 \%$ & $3.7 \%$ & $4.1 \%$ & $4.4 \%$ & $0.8 \%$ & $8.7 \%$ \\
\hline Sr & $10.6 \% *$ & $0.9 \%$ & $1.0 \%$ & $0.8 \%$ & $4.5 \%$ & $0.5 \%$ & $1.6 \% *$ \\
\hline $\mathrm{Zn}$ & $1.4 \% *$ & $0.8 \%$ & $1.8 \%$ & $0.9 \%$ & $0.2 \%$ & $1.3 \%$ & $1.5 \%$ \\
\hline
\end{tabular}

The CV has been calculated only on values above the LOQ. Values with a * indicate that the CV has been computed on less than three measurements 
rugged (namely, it shows low within-laboratory variation) $[56,57]$.

It is acknowledged that neither the standard nor the reference materials were matrix-matched. However, the exact matrix-matching the standard and the reference materials for such a large variety of complex samples (whose matrices can be very different) would have been analytically very challenging. A more generic matrixmatching by using, for instance, aged linseed oil could be a possibility for a future development of the method.

\section{Elemental characterisation of the colour tubes}

In this section, the ICP-OES results for the 57 samples, divided by colour, are shown and discussed. The results are presented as normalised stacked column histograms. Only the values above the LOQs were plotted. This presentation of the results allowed a direct comparison of the elemental content of the samples, independently of the effectiveness of the dissolution step.

The last sub-part of this section is dedicated to the presentation and discussion of the PCA of such results. As will be clearer later, this analysis allowed for an effective overview of the results on all the sampled colours.

\section{Violet}

Figure 4 shows the ICP-OES results obtained on the samples from violet paint tubes.

The substantial presence of cobalt and phosphorus in sample V1 excludes the possibility of cobalt arsenate (one of the "cobalt violets") in the manufacture of this colour, even if arsenic was not among the analysed elements [58, 59]. Supposing a homogenous dissolution of the sampled material with this method, the ratio between the quantities of cobalt and phosphorus (around 1.9) raises questions about the type of pigment employed or its purity. In fact, cobalt phosphate (the other common cobalt violet, $\left.\mathrm{Co}_{3}\left(\mathrm{PO}_{4}\right)_{2}\right)$ should give a mass ratio between cobalt and phosphorus of around 2.9. On the other hand, the fact that zinc and phosphorus stand in a mass ratio of around 1.3 may suggest the use of zinc phosphate in the manufacture (mass ratio between zinc and phosphorus of around 1.4) and the subsequent mixing with some type of cobalt salt.

Sample V6, from a paint tube without a label, contains substantial amounts of aluminium, sodium, sulfur and silicon, thus suggesting the presence of ultramarine violet. This is an alteration of ultramarine blue at high temperature and in presence of ammonium chloride [59-62].

\section{Blue}

Figure 5 shows the ICP-OES results obtained on the samples from blue paint tubes.

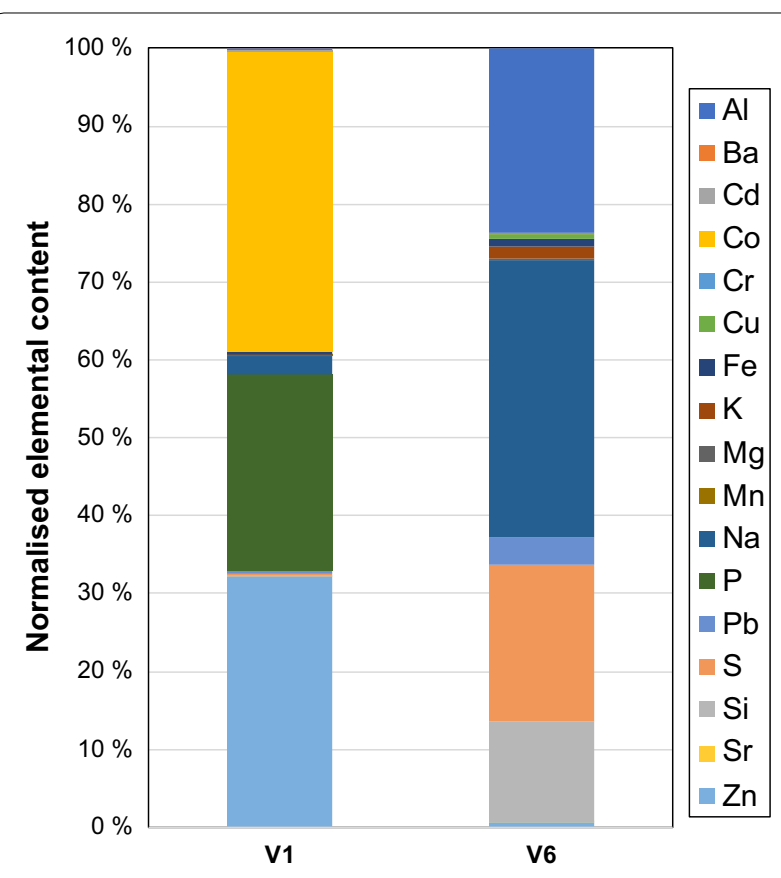

Fig. 4 Normalised elemental content of the 2 analysed violet paint tubes

Sample coming from tube B1 shows a highly diverse composition (aluminium, barium, iron, magnesium, sulfur, silicon and zinc are the major elements). Considering the nomenclature on the colour tube, indigo, the detected elements can be attributed to glass and mineral particles possibly added to improve the resistance to external chemical agents and light and the drying properties of the dye $[63,64]$, which is undetectable by this method.

Samples B7, B8, B13, B16 and B25 show a very similar composition to most lazurite-based pigments [59, 62, 65]. Magnesium (0.8-1.5\%) and iron (1.4-3.5\%), systematically present in all the samples, point to natural ultramarine as an ingredient in these tubes [66].

Samples B42 and B43 come from two tubes labelled "Cobalt blue light". Considering the high content of silicon in both samples, the relatively low content of cobalt and the overall complex elemental composition, it is reasonable to assume that both tubes contain a complex formulation of smalt $[12,59,62,67-71]$. Therefore, there must have been a shift in the production of such a colour over the years, since a modern version from the same colour from Lukas-Nerchau only contains cobalt and aluminium [72].

Samples of "Brilliant blue" (B51, B53, B55 and B56) mainly contain lead and zinc (in different proportions), pointing to the presence of lead white and zinc white, both of which were common additives to adjust the 


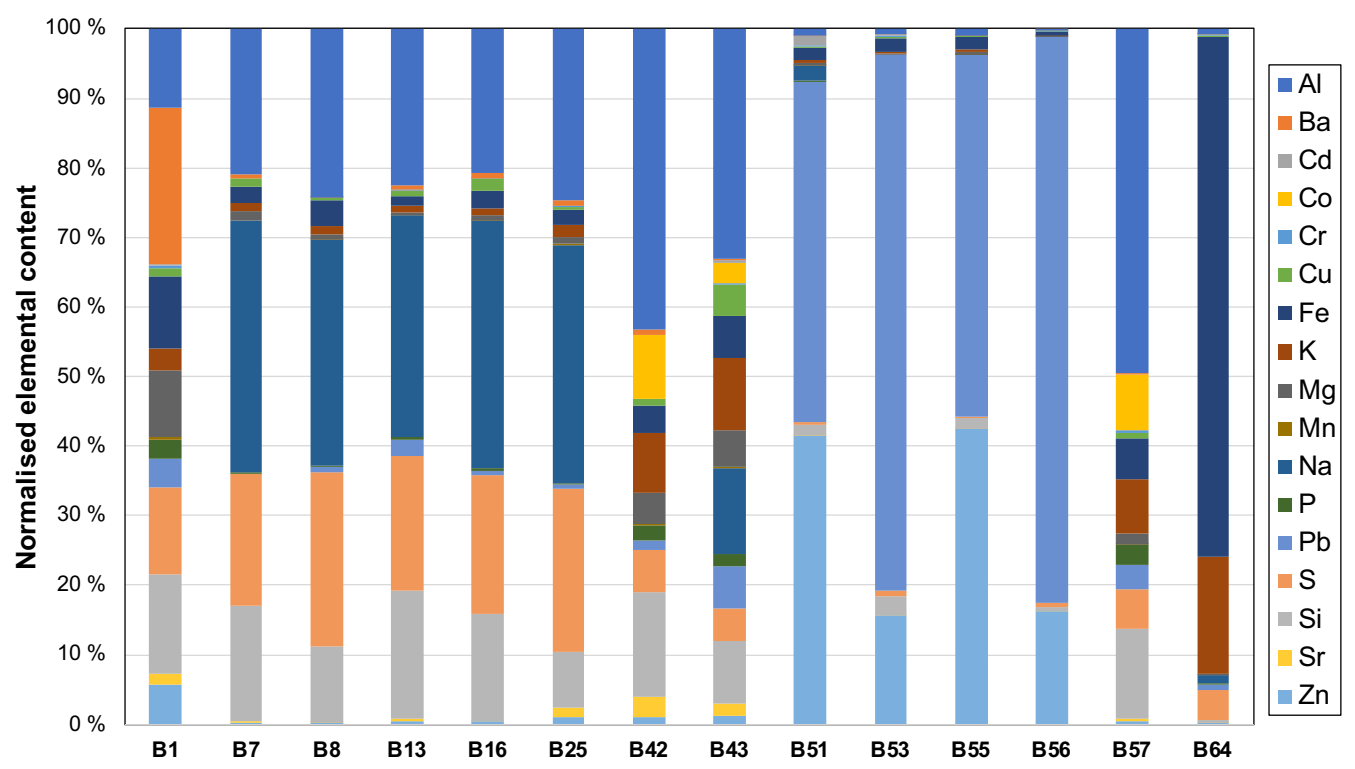

Fig. 5 Normalised elemental content of the 14 analysed blue paint tubes

vibrancy of blue paints. "Brilliant blue" is the commercial name for an indanthrene-based paint. This blue vat dye was discovered only in 1901 [59]. That Harriet Backer was using this paint so quickly thereafter demonstrates the rapid distribution of this colour and its immediate appeal to painters. In addition, two grades of Dr. Schoenfeld's Brilliant blue can probably be discerned. The lead/ zinc ratio of samples B51 and B55 (1.2) is indeed substantially different from the one of samples B53 and B56 (4.9-5.0).

Finally, the two samples coming from unlabelled paint tubes (B57 and B64) are likely those from Cobalt blue light and Prussian blue $[59,62,68]$, respectively.

\section{Green}

Figure 6 shows the ICP-OES results obtained on the samples from green paint tubes.

Samples G1, G2, G6 and G7 are from tubes labelled as "Green earth". They are rich in aluminium, iron, potassium, magnesium and silicon. Such a composition corresponds with descriptions of green earths reported in literature $[59,62,68,69,73]$, but it is worth noting that true green earth with high glauconite or celadonite content was not commonly used in oil paintings for its colour, primarily because it is transparent in oil. The colour in this instance is possibly linked to increased iron content [73]. In addition, sample G1 seems to stand out with respect to the other three because of its sodium content. However, if sodium is linked to a contamination, the relative composition of G1 with sodium removed looks consistent with the one of the other three samples.
G9, G10 and G12 are samples from tubes having "Viridian"/"Vert émeraude" labels. "Viridian" (a hydrated chromium oxide, $\mathrm{Cr}_{2} \mathrm{O}_{3} \cdot 2 \mathrm{H}_{2} \mathrm{O}$ ) was often incorrectly translated as "Vert émeraude" in French and "Verde smeraldo" in Italian $[59,62,74]$. Thus, this label is misleading. In fact, "Emerald green" in English commonly refers to copper acetate arsenite produced in different ways $[59,62,69,74]$. The presence of copper, iron, manganese and sulfur as impurities in viridian has already been reported in literature [75], although in much lower percentages. Viridian was expensive to produce $[59,75]$ and, therefore, alterations and/or unrefined production processes must have been common. The mixing with yellow barium chromate to alter the hue [59] can explain the substantial presence of barium (8.6-19.3\%) in the analyses. However, the presence of lead $(6.9-11.4 \%$ in the samples) and strontium (0.6-6.6\%) has not been previously documented. This could also be attributed to an addition of lead chromate and strontium chromate (both yellow) to make the green more vibrant.

Results for sample G15 (from an unlabelled tube) show presence of barium, chromium, iron, sodium (again, a probable impurity), lead, sulfur and silicon, leading to the conclusion that this is another viridian paint tube.

\section{Yellow}

Figure 7 shows the ICP-OES results obtained on the samples from yellow paint tubes.

The first two analysed samples, Y8 (from a "Brilliant yellow deep"-labelled paint tube) and Y9 (from a 


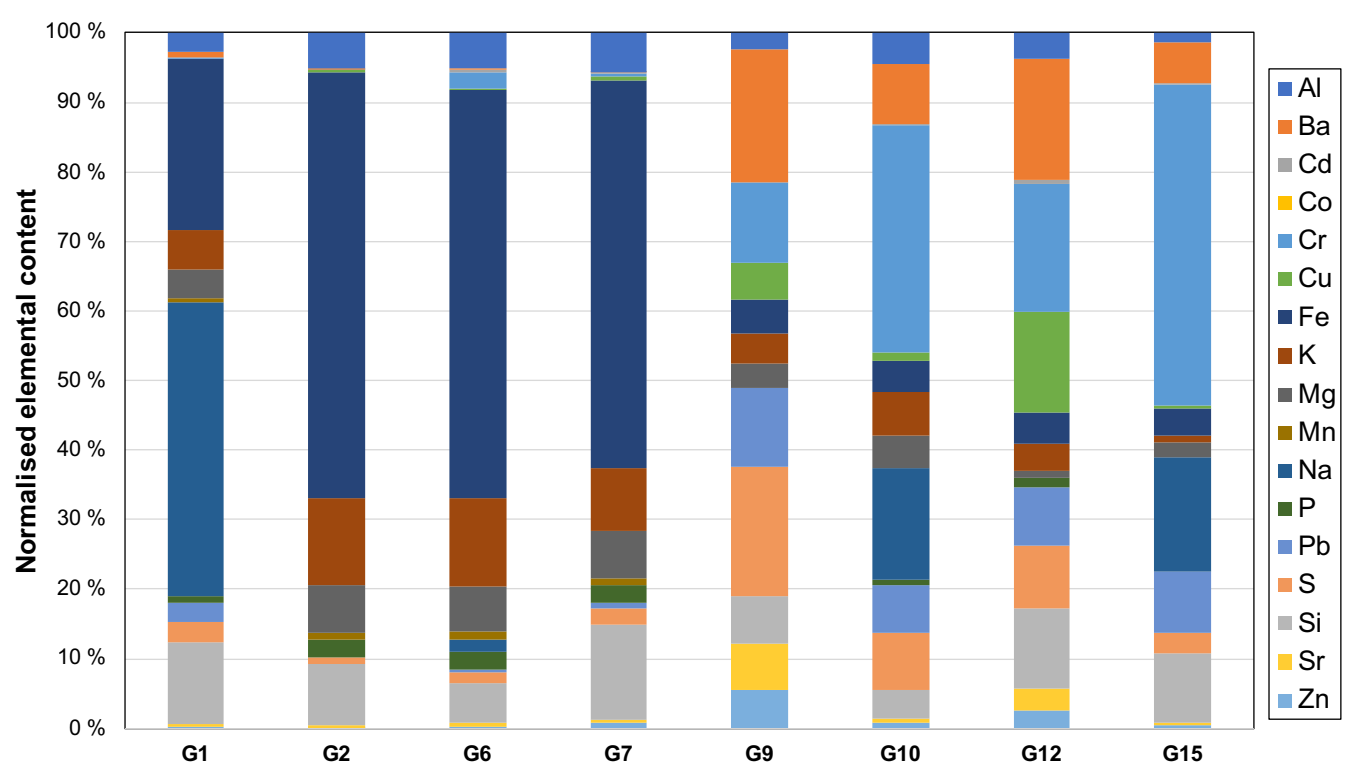

Fig. 6 Normalised elemental content of the 8 analysed green paint tubes

"Brilliant yellow light"-labelled paint tube), mainly contain lead and zinc, thus probably pointing at an organic dye as substance responsible for the colour, mixed with lead and zinc whites. This hypothesis is in clear contrast with sources that refer to "Brilliant yellow" (or, more commonly, "Jaune brilliant") as a mixture of cadmium yellow (cadmium sulfide, CdS) and zinc or lead white $[59,76-78]$. Imagining a selective dissolution that would have prevented the solubilization of cadmium is also very unlikely, considering that cadmium sulfide is readily soluble in concentrated acids $[79,80]$. Therefore, in the case of these two colours, a specific modification seems to have occurred in Dr. Schoenfeld's production.

Sample Y27 (chrome yellow) comes from the German producer C. Schmidt and it is a mixture of lead chromate with lead sulfate in molar ratio 8:6.6-7. The composition

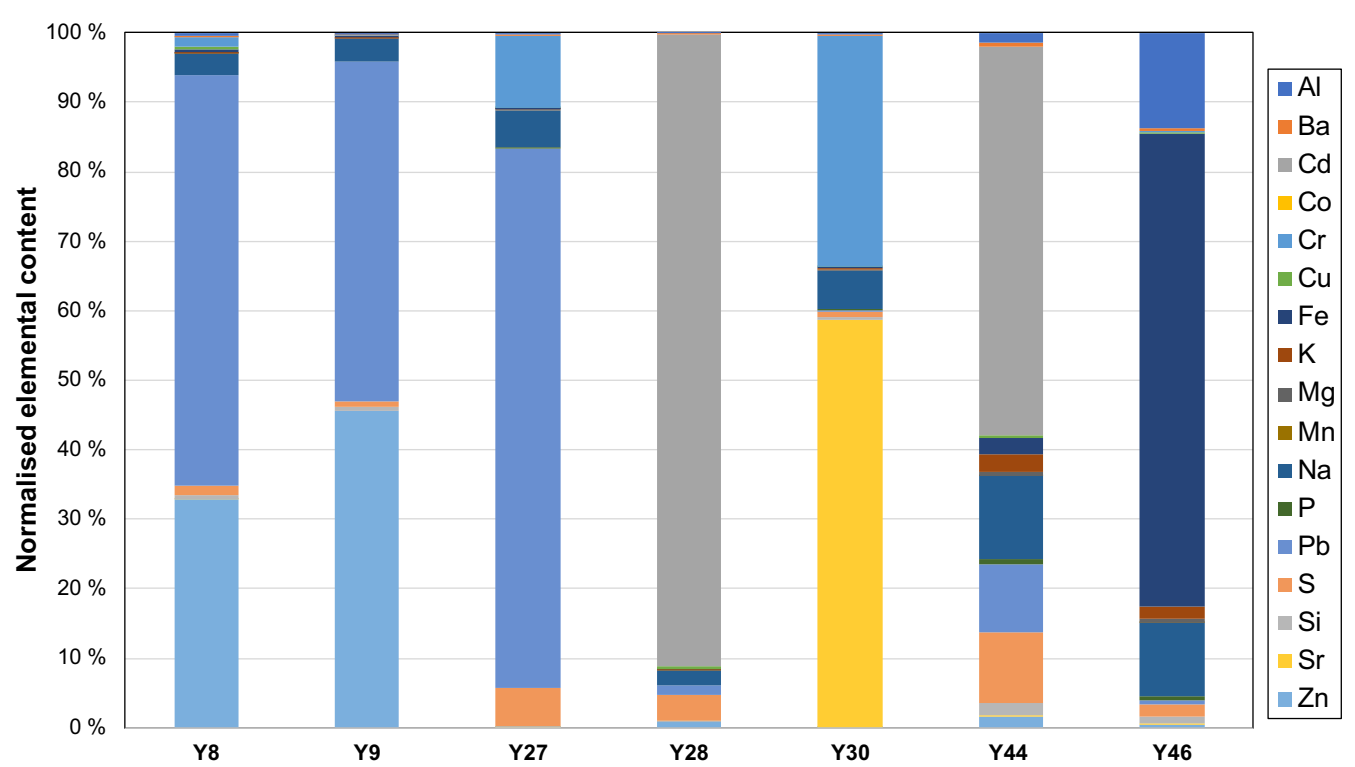

Fig. 7 Normalised elemental content of the 7 analysed yellow paint tubes 
("pale lemon" shade) is consistent with the literature on this pigment $[59,81]$.

Sample Y28 was taken from a Dr. Schoenfeld's tube without a label. Given the composition, this can be interpreted as a cadmium yellow. Still, given the content of cadmium (91.1\%) in contrast to sulfur (3.8\%), it is reasonable to think that the sulfide produced at Dr. Schoenfeld was obtained by an unrefined dry process (heating at $300-500{ }^{\circ} \mathrm{C}$ of metallic cadmium/cadmium oxide/ cadmium carbonate with elemental sulfur [78]) that left a substantial amount of unreacted cadmium/cadmium oxide/cadmium carbonate in the resulting product.

Results from sample Y30 (from another unlabelled Dr. Schoenfeld's tube) mainly show strontium and chromium in an equimolar ratio. This points to a tube containing strontium yellow, $\mathrm{SrCrO}_{4}[59,62,69,81]$.

Sample Y44, also coming from an unlabelled Dr. Schoenfeld's tube, show the presence of cadmium (55.9\%), sulfur (10.1\%) and lead (9.8\%), as main elements. Despite the incompatibility of cadmium pigments with lead ones $[59,62]$, it is difficult to imagine lead being a contaminant in this case. Y44 should therefore be a real Brilliant yellow (cadmium sulfide and lead white) [76, 78].

Finally, sample Y46 (a light-yellow ochre by the German company C. Schmidt) shows high content of iron (68.1\%) and of aluminium (13.7\%) probably indicating a yellow earth with high content of aluminium oxide/ hydroxide [82, 83].

\section{Yellowish-Red}

Figure 8 shows the ICP-OES results obtained on the samples from yellowish-red paint tubes.

Samples from Dr. Schoenfeld's paint tubes Y-R3, Y-R6, Y-R10 and Y-R11 are claimed to contain "Naples yellow reddish". This is a variety of lead antimonate [84-86]. The present ICP-OES method did not detect antimony and, therefore, it is not possible to say something about the stoichiometry of the pigment. Nevertheless, the high presence of zinc (31.2-42.5\%) in these samples seems to be peculiar to Dr. Schoenfeld's production of this colour and it has not been previously documented, if we exclude the deep yellow variety [87].

\section{Red}

Figure 9 shows the ICP-OES results obtained on the samples from red paint tubes.

Samples R3, R5, R9, R14, R15, R16 and R17 (from Dr. Schoenfeld's paint tubes labelled "Pink madder") show very similar composition, with the following elements being systematically present: aluminium (average content: $48.3 \% \pm 3.7 \%)$, phosphorus $(33.6 \% \pm 7.4 \%)$, sulfur $(6.1 \% \pm 1.1 \%)$, lead $(4.0 \% \pm 4.0 \%)$, iron $(2.8 \%+3.2 \%$, $2.8 \%-2.8 \%)$, potassium $(1.6 \% \pm 0.5 \%)$, magnesium $(0.6 \%+1.0 \%, 0.6 \%-0.6 \%)$, zinc $(0.6 \% \pm 0.5 \%)$ and barium $(0.3 \%+0.4 \%, 0.3 \%-0.3 \%)$. Phosphorus, sulfur, iron, potassium, magnesium and zinc can be ascribed to the vegetal origin of the pigment, but equally, aluminium,

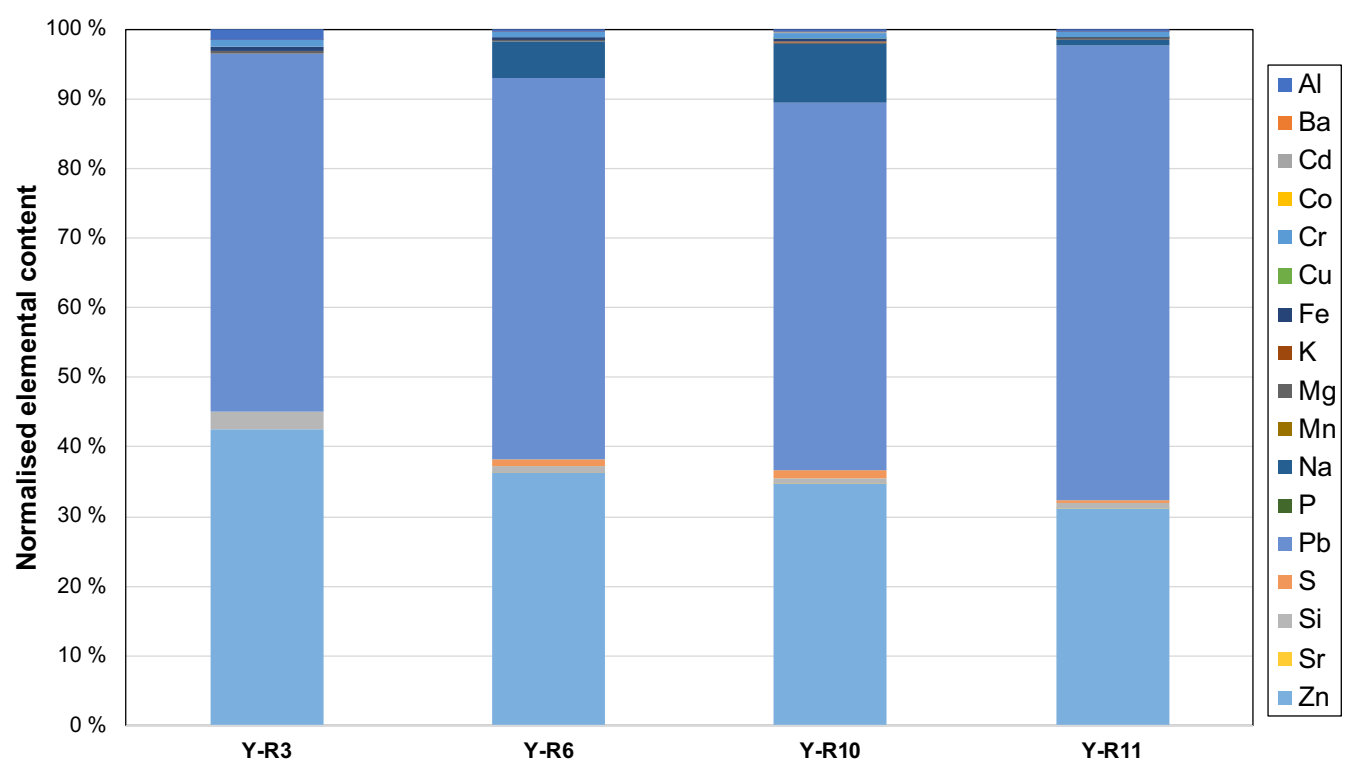

Fig. 8 Normalised elemental content of the 4 analysed yellowish-red paint tubes 


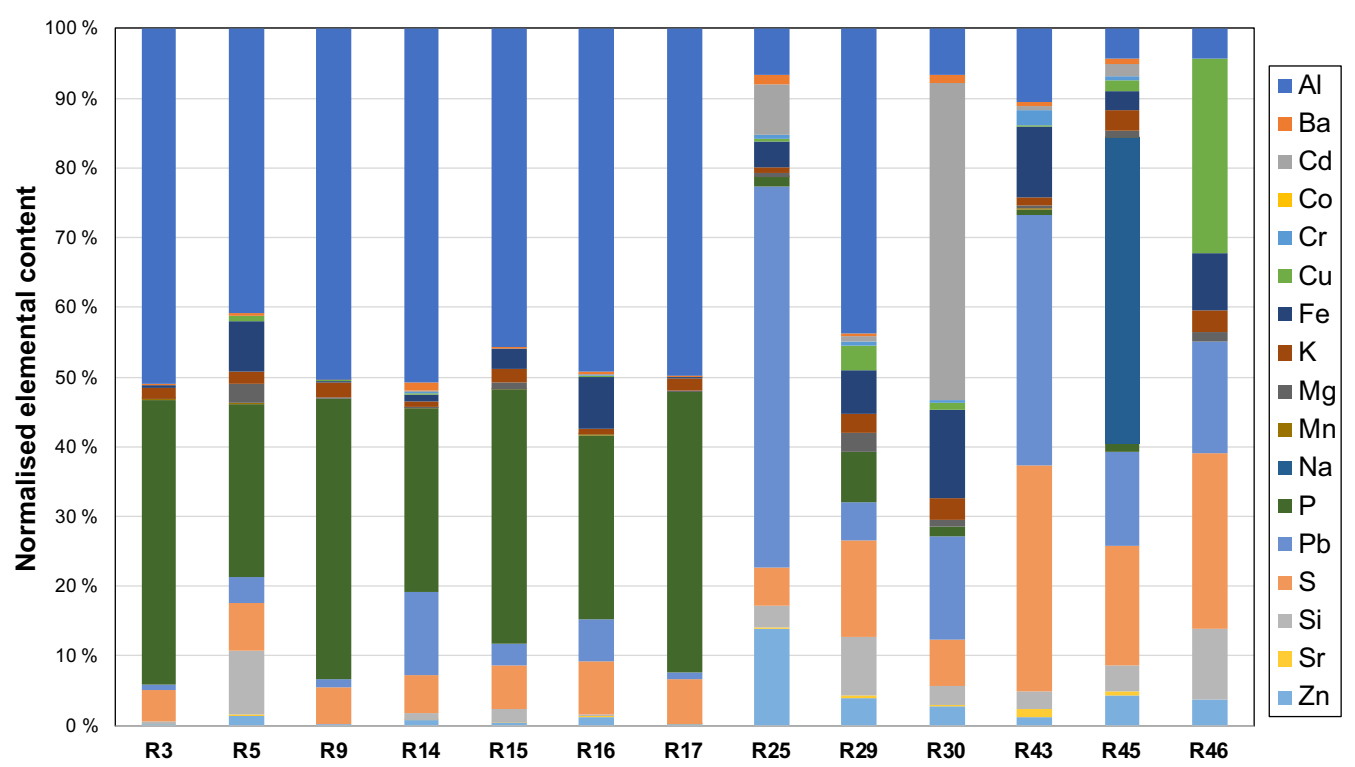

Fig. 9 Normalised elemental content of the 13 analysed red paint tubes

sulfur and potassium are likely linked to the mordant/ dye fixative $[59,88,89]$. The presence of iron is probably related to red ochre, potentially added to adjust colour and opacity [9] and lead-probably as white lead-was undoubtedly added to tune the pink hue of the resulting mixture. These results are consistent with those by XRF and EDX from the darker variant of this colour, as previously reported in the literature [11].

Samples R25, R29, R30 and R45 were taken from tubes labelled as "Finest crimson lake". They show the same qualitative composition with some relevant quantitative differences. The sample from tube R29 has the highest aluminium content (43.8\%) and has, therefore, a composition that coincides with the one reported in the literature for crimson lakes [59]. Sample R30 sticks out with its high cadmium (45.5\%) content. In this case, a hue of cadmium red could have been based on cadmium sulfide or possibly a mixture of cadmium sulfide and cadmium selenide $[59,62,69,78]$-along with the addition of an iron-based red pigment. The molar content of cadmium is, in fact, exactly double the one of sulfur. The remaining cadmium could be present as selenide but this is unlikely for at least two reasons: (i) cadmium pigments were very expensive, probably even more than the crimson ones; (ii) cadmium red, as mixture of sulfide and selenide, was not commercialized before 1910 (although its patent dates back to 1892) [59, 78] and this is incompatible with the dating of the paint box (anterior to 1909). Still, the lack of detection of selenium in this ICP-OES method makes what just exposed a speculation.
Sample R43, from a tube with a small fragment of the original label, seems to have the same qualitative composition of the samples R25, R29, R30 and R45 and can, thus, be considered from a "Finest crimson lake" colour.

Tube R46 is labelled "Chinese vermilion". The lack of detection of mercury in the method makes it hard to confirm the presence of this pigment, despite the large content of sulfur (25.0\%). Furthermore, the high content of copper (27.8\%), lead (16.0\%), silicon (10.3\%) and iron (8.2\%) corroborates the hypothesis of an adulteration. In fact, an historical source reports the use of lead oxide (minium, $2 \mathrm{PbO} \cdot \mathrm{PbO}_{2}$ ) of up to $20 \%$ in the cheaper variants of Chinese vermilion [90, 91].

\section{Brown}

Figure 10 shows the ICP-OES results obtained on the samples from brown paint tubes.

Samples Bro7 and Bro9 have compositions that coincide with those for burnt green earths and umber reported in literature $[59,73]$.

Samples Bro10, Bro11, Bro12 and Bro13 were taken from tubes without labels. They show higher lead content (probably variants) and, at the same time, lower aluminium and silicon content. They probably contained iron oxide pigments [83].

\section{White}

Figure 11 shows the ICP-OES results obtained on the samples from white paint tubes. 


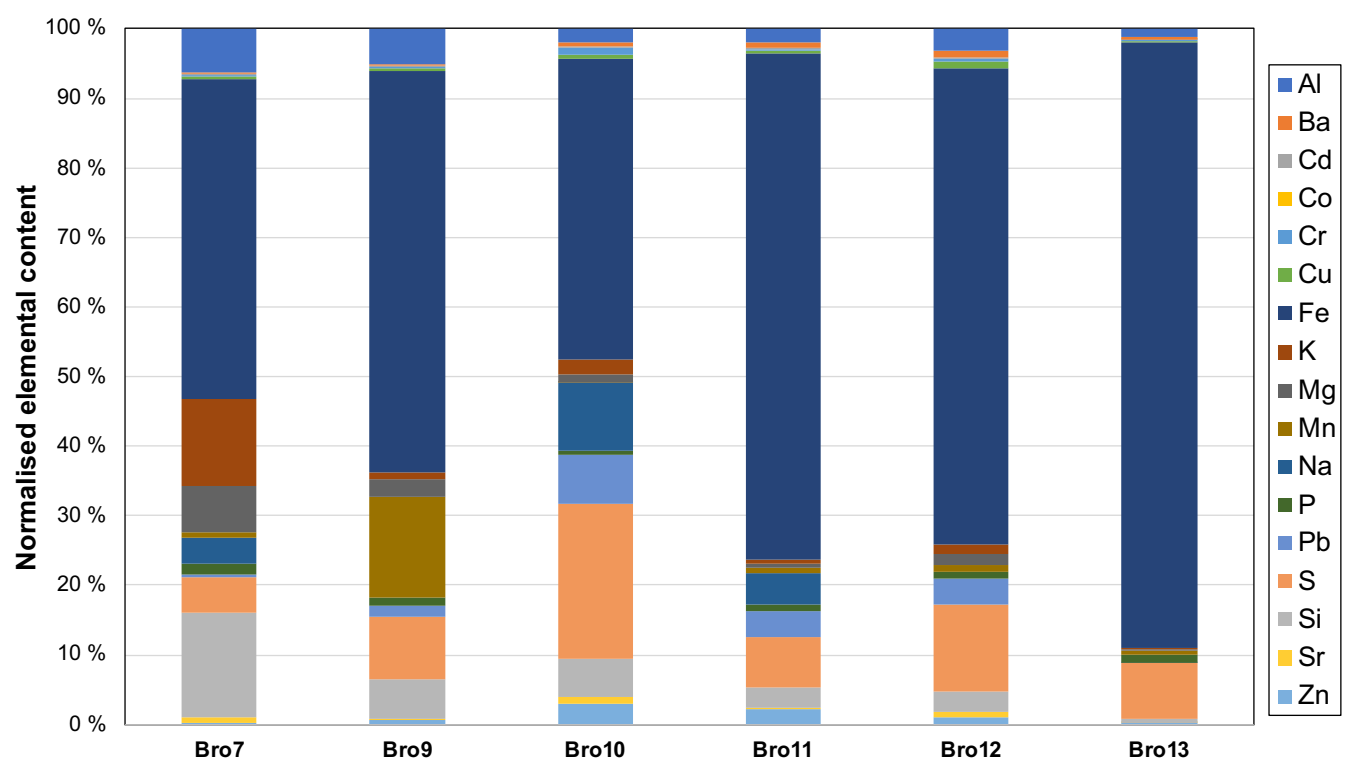

Fig. 10 Normalised elemental content of the 6 analysed brown paint tubes

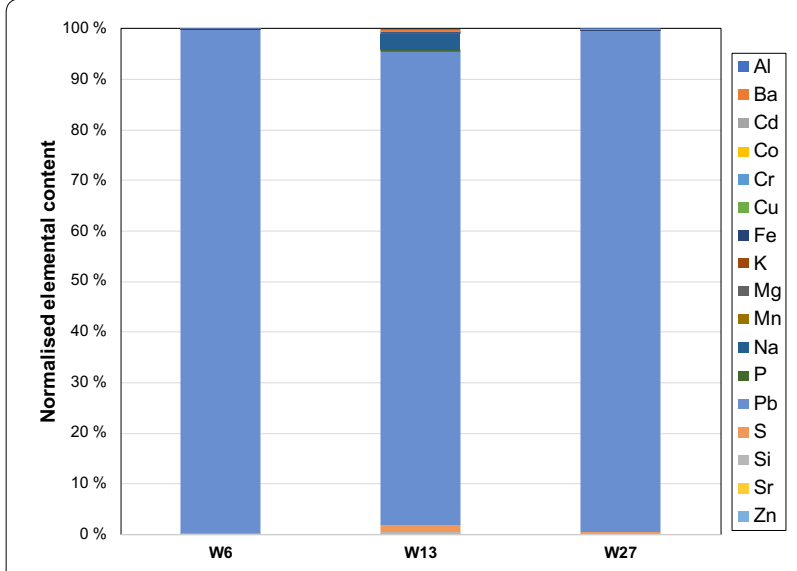

Fig. 11 Normalised elemental content of the 3 analysed white paint tubes

These are samples from three paint tubes by the Belgian producer Blockx containing highly pure lead white. Sample W13 seems to slightly stand out, but, if the possible contamination from sodium is removed, its composition is consistent with those from samples W6 and W27. It is interesting that the tubes of lead white were specifically and perhaps exclusively sourced from a Belgian firm. Makers of lead white in the Low Countries were renowned for producing lead white with the traditional Dutch stack process $[92,93]$, and several firms continued for longer than other producers in Europe up until lead white was banned from use in household paints from the 1970s. The last of the stack white manufacturers in the Netherlands closed in
1989 [94] and in Belgium, only Jeff Seynaeve continues to produce this material in small batches [92].

\section{Principal component analysis}

Figures 12 and 13 shows the results of the PCA computed on the normalised ICP-OES results from the samples from all paint tubes.

The first three PCs express a variance of $19.99 \%, 12.96 \%$ and $11.73 \%$, respectively. Although the cumulative variance of these three PCs is relatively low (and the scree plot of the PCA does not indicate any "elbow" before the tenth PC [95]), several considerations can be drawn from this multivariate analysis.

V1, B1 and Y30 stick out in both Figs. 12 and 13 because of their peculiar composition (with respect to all the other analysed samples), whereas V6 is included in the main cluster of the blues that contain aluminosilicates and smalts (B7, B8, B13, B16, B25, B42, B43 and $\mathrm{B} 57)$, confirming its production route, as suggested above in the discussion about violet colours.

G9, G10, and G12 constitute a separate group in both Figs. 12 and 13, supporting the presence of viridian (or chromium oxide [59]). The presence of what could be considered in some instances a toning agent, like Prussian blue, cannot be excluded [87]. However, the three points lie relatively far from B64 (very likely, Prussian blue) in both the principal component spaces.

The distinction of the browns can also be seen in Fig. 12, where Bro10, Bro11, Bro12 and Bro13 form a smaller group with respect to Bro7 and Bro9. G1, G2, G6 and $\mathrm{G} 7$ are close to the browns, thus demonstrating their 


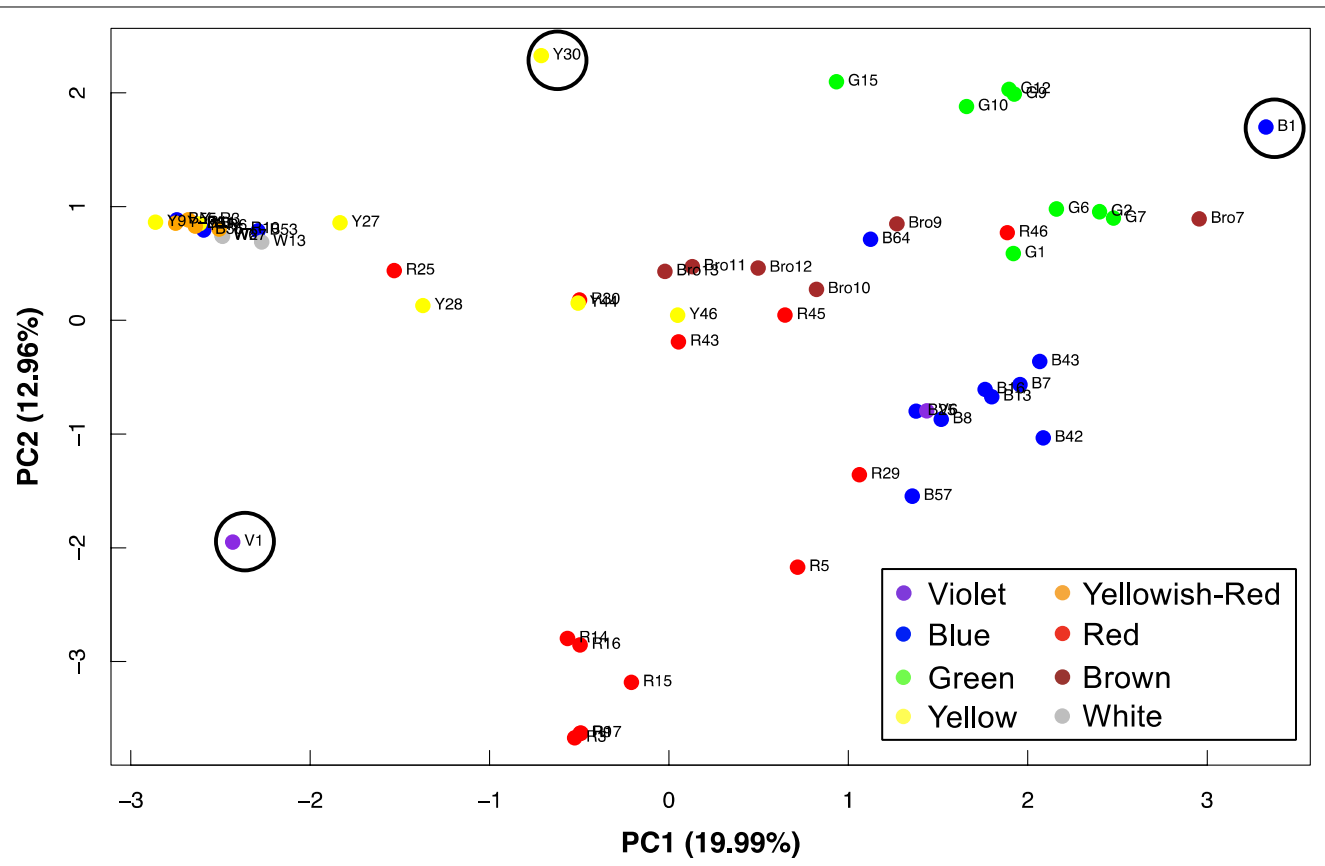

Fig. 12 Score plot of all the samples projected in the first two principal components. Samples V1, B1 and Y30 are highlighted

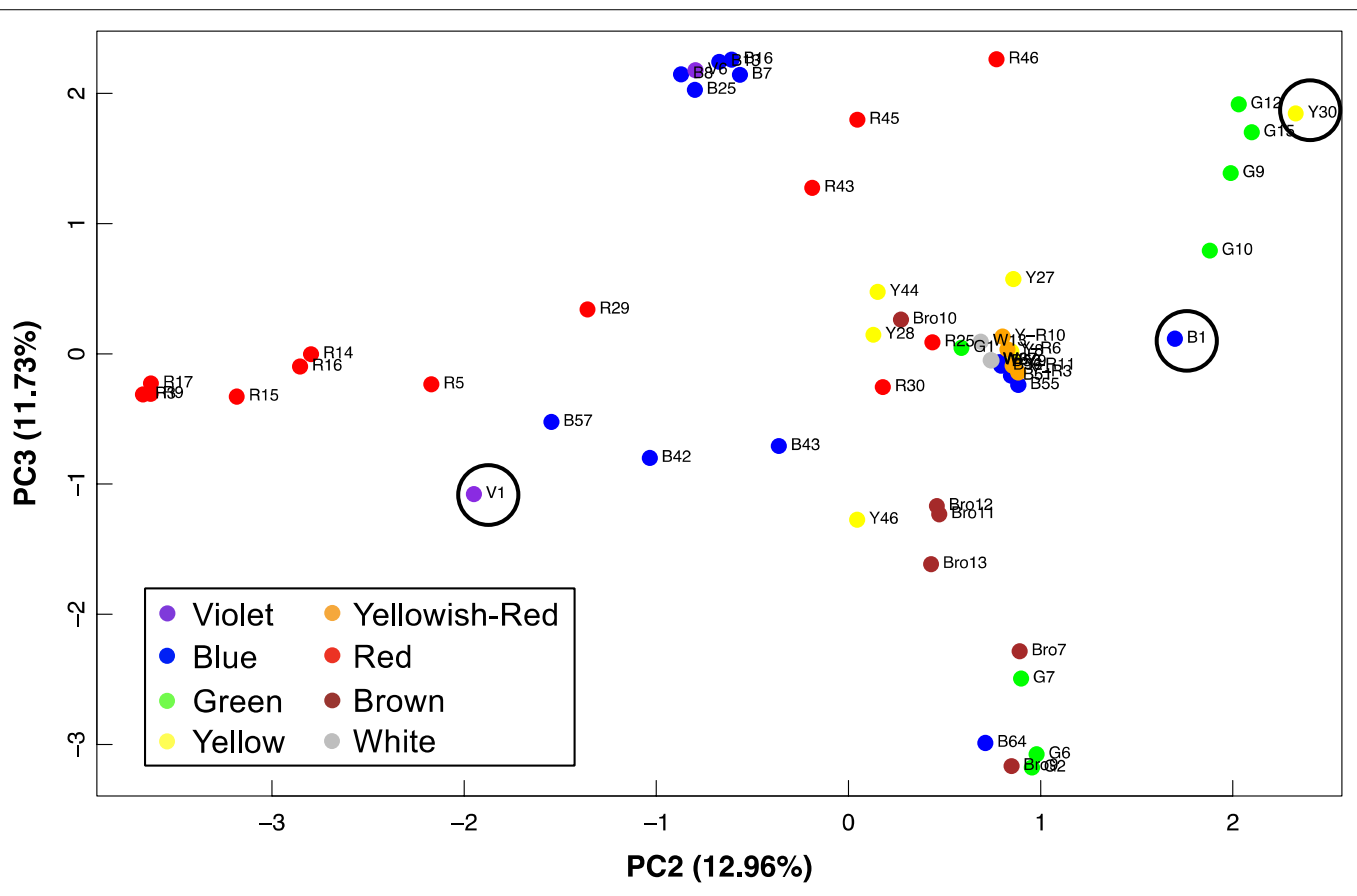

Fig. 13 Score plot of all the samples projected in the second and third principal components. Samples V1, B1 and Y30 are highlighted

earth-like nature. Although relatively isolated in Fig. 13, R46 is also in the group of the earths in Fig. 12, leading to the hypothesis of a displacement by an iron-containing compound, among others. Because of the high presence of iron (as ferric ferrocyanide), B64 is also in the large central group of Fig. 12. 
Y44 and R30 are practically superimposed in Fig. 12 and close in Fig. 13, thus corroborating the presence of cadmium sulfide in R30.

The group of the madders (R3, R5, R9, R14, R15, R16 and R17) is visible on the bottom of the Fig. 12 and on the left of Fig. 13. Sample R5 stands relatively out because of its high content of silicon.

A last large cluster is visible on the left part of Fig. 12 and at the centre of Fig. 13. Such a cluster contains those samples with high concentrations of lead and zinc whites: all the yellowish-reds, all the whites, the four Brilliant blues (B51, B53, B55 and B56) and the two Brilliant yellows (Y8 and Y9). In the case of the yellowish-red colours, only the presence of antimony could rule out another production modification.

The performed PCA allowed the visualization of the compositional results of 57 samples in one-two plots, readily identifying clusters and outliers among the samples. The score plots of Figs. 12 and 13 can be thus considered complementary view modes for the results previously shown by the histograms.

\section{Conclusions}

This work has presented a novel and validated ICP-OES method for the quantitative analysis of 17 elements in micro-samples $(4 \mathrm{mg} \pm 3 \mathrm{mg}$ ) from historical paint tubes. When compared to an ISO method on a relatively similar matrix, this method requires very little in terms of quantity of sample, pushing the limits for the elemental analysis of complex organic-based matrices. Although, in general, developments of ICP-OES methods are timeconsuming and entail significant laboratory work, this approach provides the user with very accurate elemental bulk characterization not achievable by other techniques such as EDX or XRF. Our method has proven to be accurate and rugged and has successfully provided, for the first time, the quantitative elemental characterisation of samples from 57 paint tubes contained in the paint box belonged to the Norwegian female painter, Harriet Backer. Furthermore, most of the tubes were produced by the German company Dr. Schoenfeld \& Co. and, as such, several interesting aspects have been brought to light related to their production between the end of 19th century and the beginning of 20th century (for example, in compositions of brilliant yellows and the finest crimson lakes). Despite the apparently low variance (the cumulative one on the first three PCs is equal to $44.68 \%$ ), the multivariate analysis supported well these results, which in their entirety will constitute a useful reference when other scientific analyses are performed on Harriet Backer's paintings.

Although already very powerful, as an outlook for the future, this ICP-OES method could be improved by detecting and quantifying antimony, arsenic, boron, calcium, mercury, selenium and tin; and by preparing matrix-matched standard and reference solutions (e.g., by using artificially or naturally aged linseed oil).

Considering the quality of the data obtained during this study, the resulting wealth of information on the materials and the relatively low quantity of sample, this study has the ambition to pave the way for ICP-OES to become an established method in the heritage science community when analysing historical paint materials.

\section{Additional file}

Additional file 1. Catalogue of Harriet Backer's 259 colour tubes in Uvdal stave church.

\section{Authors' contributions}

FC, SM and TF designed the research. FC and SM performed the experiments. All authors contributed in analysing the data and writing the paper. All authors read and approved the final manuscript.

\section{Author details}

${ }^{1}$ Institutt for arkeologi, konservering og historie (IAKH), Conservation Studies, Universitetet i Oslo (UiO), Postboks 1008, Blindern, 0315 Oslo, Norway. ${ }^{2}$ Institut für Baustoffe (IfB), Physical Chemistry of Building Materials (PCBM), ETH Zürich, Stefano-Franscini-Platz 3, HIF B64.2, 8093 Zurich, Switzerland.

\section{Acknowledgements}

The authors are grateful to Merete Winness from Fortidsminneforeningen for access to the paint tubes and to KODE Arts Museums in Bergen for the photos of their easel paintings from Uvdal stave church. The authors wish to thank Ingo Sprenger from Lukas-Nerchau GmbH for the precious information given about the historical production aspects of the paint tubes. The authors are indebted to Ingvild Vestvik for photo-documentation of Harriet Backer's paint tubes, during her time as research assistant at Conservation Studies, IAKH-UiO. The authors also would like to thank Hartmut Kutzke (Museum of Cultural History, $\mathrm{UiO}$ ) for the insightful discussions about the work of this paper.

\section{Competing interests}

The authors declare that they have no competing interests.

\section{Availability of data and materials}

Raw data and high-quality images are available from the authors on request.

\section{Funding}

Not applicable.

\section{Publisher's Note}

Springer Nature remains neutral with regard to jurisdictional claims in published maps and institutional affiliations.

Received: 2 November 2018 Accepted: 24 December 2018

Published online: 07 January 2019

\section{References}

1. Lange M. Harriet Backer. Oslo: Gyldendal; 1995

2. Kutzke H, Frøysaker T. Harriet Backer: the artist's paint tubes in Uvdal stave church and her corresponding paintings. In: Krekel C, Townsend JH, Eyb-Green S, Pilz K, editors. Expr Sensib-art technol sources rise mod. London: Archetype Publications Ltd; 2018. p. 141-2. 
3. Fortidsminneforeningen. Uvdal stavkirke; 2018. https://www.fortidsmin neforeningen.no/vare-eiendommer/uvdal-stavkirke. Accessed 30 Aug 2018.

4. Fortidsminneforeningen. Harriet Backers malesaker; 2018. https://www. fortidsminneforeningen.no/digitalt-museum/harriet-backers-malesaker Accessed 30 Aug 2018.

5. Rand JG. Improvement in the construction of vessels or apparatus for preserving paint; 1841. https://patents.google.com/patent/US2252A/en? $q=j o h n \& q=$ rand $\& o q=j o h n+$ rand. Accessed 3 Sept 2018.

6. Hurt P. Never Underestimate the Power of a Paint Tube. Smithson. Mag; 2013. https://www.smithsonianmag.com/arts-culture/never-underestim ate-the-power-of-a-paint-tube-36637764/. Accessed 3 Sept 2018.

7. Bird M. A real squeeze: paint in tubes; 2015. https://www.christies.com/ features/14-Art-Media-Paint-in-Tubes-5840-1.aspx. Accessed 3 Sept 2018.

8. Winsor and Newton. From the archives: the history of the metal paint tube; 2015. http://www.winsornewton.com/row/discover/articles-andinspiration/from-the-archives-history-of-thet-metal-paint-tube. Accessed 3 Sept 2018.

9. Townsend JH, Carlyle L, Khandekar N, Woodcock S. Later nineteenth century pigments: evidence for additions and substitutions. Conservator. 1995;19:65-78.

10. Van De Laar M, Burnstock A. "With Paint from Claus \& Fritz": a Study of an Amsterdam Painting Materials Firm (1841-1931). J Am Inst Conserv. 1997:36:1-16.

11. Diependaal $H$, van den Berg KJ, de Groot S, de Keijzer M, van Keulen H, Megens L. Breitner's paint box and the colours of Standage. Z Für Kunsttechnol Konserv. 2013;27:171-91.

12. Izzo FC, van den Berg KJ, van Keulen H, Ferriani B, Zendri E. Modern oil paints - formulations, organic additives and degradation: some case studies. In: van den Berg KJ, Burnstock A, de Keijzer M, Krueger J, Learner T, de Alberto Tagle, editors. Issues contemp oil paint. Cham: Springer International Publishing; 2014. p. 75-104. https://doi.org/10.1007/978-3319-10100-2_5.

13. Pitthard V, Stanek S, Griesser M, Muxeneder T. Gas chromatography-mass spectrometry of binding media from early 20th century paint samples from Arnold Schönberg's Palette. Chromatographia. 2005;62:175-82.

14. Cruz AJ. A pintura de Columbano Segundo as suas Caixas de Tintas e Pincéis. Conserv Património. 2005;1:5-19.

15. Montagner C, Sanches D, Pedroso J, Melo MJ, Vilarigues M. Ochres and earths: matrix and chromophores characterization of 19th and 20th century artist materials. Spectrochim Acta A Mol Biomol Spectrosc. 2013;103:409-16.

16. FitzHugh EW, Leona M, Shibayama N. Pigments in a paint box belonging to whistler in the library of congress. Stud Conserv. 2011;56:115-24.

17. Wachowiak M. 19th century paints of Richard Ainè used by Jan Matejko (1838-1983). Analysis of preserved paints from tubes, palettes and of paintings' surfaces and paint-layer. In: Radvan R, Asmus JF, Castillejo M, Pouli P, Nevin A, editors. Lasers Conserv Artworks VIII. Leiden: CRC Press; 2011. p. 173-6.

18. Otłowska O, Ślebioda M, Wachowiak M, Śliwka-Kaszyńska M. Identification and characterization of the Indian Yellow dyestuff and its degradation products in historical oil paint tube by liquid chromatography mass spectrometry. RSC Adv. 2015;5:48786-92.

19. Monico L, Van der Snickt G, Janssens K, De NolfW, Miliani C, Verbeeck $J$, et al. Degradation process of lead chromate in paintings by Vincent van Gogh studied by means of synchrotron X-ray spectromicroscopy and related methods. 1. Artificially aged model samples. Anal Chem. 2011:83:1214-23.

20. Monico L, Van der Snickt G, Janssens K, De NolfW, Miliani C, Dik J, et al. Degradation process of lead chromate in paintings by Vincent van Gogh studied by means of synchrotron X-ray spectromicroscopy and related methods. 2. Original paint layer samples. Anal Chem. 2011;83:1224-31.

21. Monico L, Janssens K, Miliani C, Brunetti BG, Vagnini M, Vanmeert F, et al. Degradation process of lead chromate in paintings by Vincent van Gogh studied by means of spectromicroscopic methods. 3. Synthesis, characterization, and detection of different crystal forms of the chrome yellow pigment. Anal Chem. 2013;85:851-9.

22. Monico L, Janssens K, Miliani C, Van der Snickt G, Brunetti BG, Cestelli Guidi $M$, et al. Degradation process of lead chromate in paintings by Vincent van Gogh studied by means of spectromicroscopic methods. 4
Artificial aging of model samples of co-precipitates of lead chromate and lead sulfate. Anal Chem. 2013;85:860-7.

23. Monico L, Janssens K, Vanmeert F, Cotte M, Brunetti BG, Van der Snickt $G$, et al. Degradation process of lead chromate in paintings by vincent van gogh studied by means of spectromicroscopic methods. Part 5. Effects of nonoriginal surface coatings into the nature and distribution of chromium and sulfur species in chrome yellow paints. Anal Chem. 2014;86:10804-11.

24. Monico L, Janssens $K$, Hendriks E, Vanmeert F, Van der Snickt G, Cotte $M$, et al. Evidence for degradation of the chrome yellows in Van Gogh's sunflowers: a study using noninvasive in situ methods and synchrotronradiation-based X-ray techniques. Angew Chem Int Ed. 2015;54:13923-7.

25. Janssens K, Van der Snickt G, Vanmeert F, Legrand S, Nuyts G, Alfeld M, et al. Non-invasive and non-destructive examination of artistic pigments, paints, and paintings by means of X-ray methods. In: Mazzeo R, editor. Anal Chem Cult Herit. Cham: Springer International Publishing; 2017. p. 77-128. https://doi.org/10.1007/978-3-319-52804-5_3.

26. Otero V, Carlyle L, Vilarigues M, Melo MJ. Chrome yellow in nineteenth century art: historic reconstructions of an artists' pigment. RSC Adv. 2012:2:1798-805.

27. Otero V, Pinto JV, Carlyle L, Vilarigues M, Cotte M, Melo MJ. Nineteenth century chrome yellow and chrome deep from Winsor \& Newton ${ }^{\text {TM }}$. Stud Conserv. 2017;62:123-49.

28. Munchmuseet. Edvard Munch's paint tubes and working materials. Munchmuseet. 2018. https://munchmuseet.no/en/conservation-munch s-paint-tubes-and-working-materials. Accessed 5 Sept 2018.

29. Kutzke H, Topalova-Casadiego B. Munch's colour tubes: a hidden treasure at the Munch Museu, Oslo. In: Frøysaker T, Streeton N, Kutzke H, HanssenBauer F, Topalova-Casadiego B, editors. Public paint Edvard Munch his contemp_change conserv chall. London: Archetype Publications Ltd; 2015. p. 204-10.

30. Uldanck D, Zanaboni M, Modugno F, Colombini MP, Kutzke H, TopalovaCasadiego B. Munch's colour tubes: analysis of binding media. In: Frøysaker T, Streeton N, Kutzke H, Hanssen-Bauer F, Topalova-Casadiego B, editors. Public paint Edvard Munch his contemp-change conserv chall. London: Archetype Publications Ltd; 2015. p. 211-6.

31. La Nasa J, Zanaboni M, Uldanck D, Degano I, Modugno F, Kutzke H, et al. Novel application of liquid chromatography/mass spectrometry for the characterization of drying oils in art: elucidation on the composition of original paint materials used by Edvard Munch (1863-1944). Anal Chim Acta. 2015;896:177-89.

32. Klempan B, Corbeil MC, Poulin J, Cook P. A technical and scientific study of two A.Y. Jackson paintboxes. J Can Assoc Conserv. 2009;34:29-38.

33. Christiansen MB, Baadsgaard E, Sanyova J, Simonsen KP. The artists' materials of P. S. Krøyer: An analytical study of the artist's paintings and tube colours by Raman, SEM-EDS and HPLC. Herit Sci. 2017;5:39.

34. Christiansen MB, Sørensen MA, Sanyova J, Bendix J, Simonsen KP. Characterisation of the rare cadmium chromate pigment in a 19th century tube colour by Raman, FTIR, X-ray and EPR. Spectrochim Acta A Mol Biomol Spectrosc. 2017;175:208-14.

35. Rogge CE, Epley BA. Behind the Bocour Label: identification of pigments and binders in historic bocour oil and acrylic paints. J Am Inst Conserv. 2017:56:15-42.

36. Lee JA, Ormsby BA, Burnstock A, Schilling M, van den Berg KJ. The chemical characterisation of water-sensitive oil paint swatches by Winsor \& Newton. In: Bridgland J, editor. ICOM-CC 18th Triennial Conference Preprints Copenhagen 4-8 Sept 2017. Paris: International Council of Museums; 2017. p. art. 1604

37. Lee J, Bonaduce I, Modugno F, La Nasa J, Ormsby B, van den Berg KJ. Scientific investigation into the water sensitivity of twentieth century oil paints. Microchem J. 2018;138:282-95.

38. Plahter U. Verdien av maletekniske undersøkelser-forslag til rutiner. In: Stein M, Gundhus G, Johannesen NH, editors. Kirkekunsten Lider Hvordan Bevare Middelalderkunsten Nor Kirker. Øvre Ervik: Alvheim \& Eide; 1987. p. $45-7$.

39. ISO/TC 238 Solid biofuels. ISO 16967:2015-Solid biofuels-determination of major elements-Al, Ca, Fe, Mg, P, K, Si, Na and Ti; 2015. https:// www.iso.org/standard/58065.html. Accessed 5 Sept 2018.

40. Caruso F, Mantellato S, Palacios M, Flatt RJ. ICP-OES method for the characterization of cement pore solutions and their modification by polycarboxylate-based superplasticizers. Cem Concr Res. 2017:91:52-60. 
41. Bener A, Lovric M. Variance. In: Lovric M, editor. Int Encycl Stat Sci. Berlin: Springer; 2011. p. 1640-2. https://doi.org/10.1007/978-3-642-04898 -2_634. Accessed 2 Oct 2018.

42. Wold S, Esbensen K, Geladi P. Principal component analysis. Chemom Intell Lab Syst. 1987;2:37-52.

43. Ringnér M. What is principal component analysis? Nat Biotechnol 2008;26:303-4.

44. Miller JN, Miller JC. Statistics and chemometrics for analytical chemistry. 6th ed. Harlow: Pearson Education Ltd.; 2010.

45. Jolliffe I. Principal component analysis. In: Lovric M, editor. Int Encycl Stat Sci. Berlin: Springer; 2011. p. 1094-6. https://doi.org/10.1007/978-3-64204898-2_455. Accessed 30 Sept 2018.

46. Bro R, Smilde AK. Principal component analysis. Anal Methods. 2014;6:2812-31

47. R Core Team. R: A language and environment for statistical computing. Vienna: R Foundation for Statistical Computing; 2018. https://www.Rproject.org/. Accessed 26 Dec 2018.

48. RStudio Team. RStudio: Integrated Development for R. Boston: RStudio, Inc.; 2017. http://www.rstudio.com/. Accessed 26 Dec 2018.

49. ISO/TC 69/SC 6 Measurement methods and results. ISO 5725-1:1994accuracy (trueness and precision) of measurement methods and results_-part 1: general principles and definitions; 1994. http://www. iso.org/iso/home/store/catalogue_ics/catalogue_detail_ics.htm?csnum ber $=11833$. Accessed 26 Dec 2018.

50. Nič M, Jirát J, Košata B, Jenkins A, McNaught $A$, editors. Limit of detection in analysis. IUPAC Compend Chem Terminol. 2.1.0. Research Triagle Park, NC: IUPAC; 2009. http://goldbook.iupac.org/L03540.html. Accessed 4 Aug 2015.

51. Uhrovčík J. Strategy for determination of LOD and LOQ values-some basic aspects. Talanta. 2014;119:178-80.

52. Nič $M$, Jirát J, Košata $B$, Jenkins $A$, McNaught $A$, editors. precision. IUPAC Compend Chem Terminol. 2.1.0. Research Triagle Park, NC: IUPAC; 2009. http://goldbook.iupac.org/P04799.html. Accessed 19 Nov 2015.

53. Fitzsimmons JM, Medvedev DG, Mausner LF. Specific activity and isotope abundances of strontium in purified strontium-82. J Anal At Spectrom. 2016;31:458-63.

54. Boss CB, Fredeen KJ. Concepts, instrumentation and techniques in inductively coupled plasma optical emission spectrometry. 3rd ed. Waltham: Perkin-Elmer; 2004.

55. Hill SJ, Fisher A, Foulkes M. Basic concepts and instrumentation for plasma spectrometry. In: Hill SJ, editor. Inductively coupled plasma spectrometry its applications. 2nd ed. Hoboken: Blackwell Publishing Ltd; 2007

56. Aguilera E, Lucena R, Cárdenas S, Valcárcel M, Trullols E, Ruisánchez I. Robustness in qualitative analysis: a practical approach. TrAC Trends Anal Chem. 2006:25:621-7.

57. Ferreira SLC, Caires AO, da Borges ST, Lima AMDS, Silva LOB, dos Santos WNL. Robustness evaluation in analytical methods optimized using experimental designs. Microchem J. 2017;131:163-9.

58. Corbeil M-C, Charland J-P, Moffatt EA. The characterization of cobalt violet pigments. Stud Conserv. 2002;47:237-49.

59. Eastaugh N, Walsh V, Chaplin T, Siddall R. Pigment compendium-a dictionary and optical microscopy of historical pigments. Oxford: Butterworth-Heinemann; 2008.

60. Seel F. Sulfur in artwork: Lapis Lazuli and ultramarine pigments. In: Müller A, Krebs B, editors. Stud inorg chem. Elsevier; 1984. p. 67-89. http://www. sciencedirect.com/science/article/pii/B9780444423559500098. Accessed 15 Sept 2018

61. FitzHugh EW, Zycherman LA. A purple barium copper silicate pigment from early China. Stud Conserv. 1992;37:145-54.

62. Matteini M, Mazzeo R, Moles A. Chemistry for Restoration. Florence: Nardini; 2017

63. Lutzenberger $\mathrm{K}$, Stege $\mathrm{H}$, Tilenschi $\mathrm{C}$. A note on glass and silica in oil paintings from the 15th to the 17th century. J Cult Herit. 2010;11:365-72.

64. Dejoie C, Martinetto P, Dooryhée E, Elslande EV, Blanc S, Bordat P, et al. Association of indigo with zeolites for improved color stabilization. Appl Spectrosc. 2010;64:1131-8.

65. Fleet ME, Liu X. X-ray absorption spectroscopy of ultramarine pigments: a new analytical method for the polysulfide radical anion S3-chromophore. Spectrochim Acta Part B At Spectrosc. 2010;65:75-9.
66. Osticioli I, Mendes NFC, Nevin A, Gil FPSC, Becucci M, Castellucci E. Analysis of natural and artificial ultramarine blue pigments using laser induced breakdown and pulsed Raman spectroscopy, statistical analysis and light microscopy. Spectrochim Acta A Mol Biomol Spectrosc. 2009;73:525-31.

67. Fukunaga K. THz Spectroscopy. In: Fukunaga K, editor. THz Technol Appl Cult Herit Pract. Tokyo: Springer Japan; 2016. p. 23-45. https://doi. org/10.1007/978-4-431-55885-9 3. Accessed 23 Sept 2018.

68. Harley RD. Artists' Pigments C 1600-1835-a study in english documentary sources, 2nd revised. London: Archetype Publications Ltd.; 2001.

69. Casoli A, Colombini MP, Matteini M. La chimica dei materiali pittorici. In: Campanella L, editor. Chim Arte. Bologna: Zanichelli; 2007. p. 43-156.

70. Mühlethaler B, Thissen J. Smalt. In: Roy A, editor. Artists pigments—A handbook of their history and character. Washington: National Gallery of Art; 1993. p. 113-30.

71. Jonynaitè D, Senvaitienè J, Kiuberis J, Kareiva A, Juškènas R, Ramanauskas R. XRD characterization of cobalt-based historical pigments and glazes. Chemija. 2009;20:10-8.

72. Vila A, Ferrer N, García JF. Colored inks analysis and differentiation: a first step in artistic contemporary prints discrimination. Anal Chim Acta. 2007:588:96-107.

73. Grissom CA. Green Earth. In: Feller RL, editor. Artists pigments—A handbook of their history and character. Washington: National Gallery of Art; 1986. p. 141-67.

74. Fiedler I, Bayard MA. Emerald Green and Scheele's Green. In: FitzHugh EW, editor. Artists pigments - A handbook of their history and character. Washington: National Gallery of Art; 1997. p. 219-71.

75. Newman R. Chromium oxide greens_chromium oxide and hydrated chromium oxide. In: FitzHugh EW, editor. Artists pigments-A handbook of their history and character. Washington: National Gallery of Art; 1997. p. 273-93.

76. Doerner M. The materials of the artist and their use in painting with notes on the techniques of the old masters. New York: Harcourt, Brace and Company, Inc.; 1949.

77. Müller HG. Schoenfeld's malerfibel_-pigmente und bindemittel. Düsseldorf: Schwann; 1962

78. Fiedler I, Bayard MA. Cadmium yellows, oranges and reds. In: Feller RL, editor. Artists pigments-A handbook of their history and character. Washington: National Gallery of Art; 1986. p. 65-108.

79. Vogel Al. Vogel's qualitative inorganic analysis. 7th ed. Harlow: Longman; 1997.

80. Van der Snickt G, Dik J, Cotte M, Janssens K, Jaroszewicz J, De Nolf W, et al. Characterization of a degraded cadmium yellow (CdS) pigment in an oil painting by means of synchrotron radiation based X-ray techniques. Anal Chem. 2009:81:2600-10.

81. Kühn H, Curran M. Chrome Yellow and other chromate pigments. In: Feller RL, editor. Artists pigments—A handbook of their history and character. Washington: National Gallery of Art; 1986. p. 187-217.

82. Helwig K. The characterisation of iron earth pigments using infrared spectroscopy. Postprints IRUG2 VA. London: Victoria \& Albert Museum; 1998. p. 83-92.

83. Helwig K. Iron oxide pigments—natural and synthetic. In: Berrie BH editor. Artists pigments-A handbook of their history and character. Washington: National Gallery of Art; 2007. p. 39-109.

84. Wainwright INM, Taylor JM, Harley RD. Lead antimonate yellow. In: Feller $\mathrm{RL}$, editor. Artists pigments-A handbook of their history and character. Washington: National Gallery of Art; 1986. p. 219-54.

85. Dik J, Tichelaar F, Goubitz K, Peschar R, Schenk H. 19th Century Naples yellow re-examined. Z Für Kunsttechnol Konserv. 2002;16:291-306.

86. Hradil D, Grygar T, Hradilová J, Bezdička P, Grűnwaldová V, Fogaš I, et al. Microanalytical identification of $\mathrm{Pb}-\mathrm{Sb}$-Sn yellow pigment in historical European paintings and its differentiation from lead tin and Naples yellows. J Cult Herit. 2007:8:377-86.

87. Richter EL, Härlin H. The pigments of the swiss nineteenth-century painter Arnold Böcklin. Stud Conserv. 1974;19:83-7.

88. Kirby J, Spring M, Higgitt C. The technology of red lake pigment manufacture: study of the Dyestuff Substrate. Natl Gallery Tech Bull. 2005; $26: 71-87$

89. Pozzi F, Lombardi JR, Leona M. Winsor \& Newton original handbooks: a surface-enhanced Raman scattering (SERS) and Raman spectral database of dyes from modern watercolor pigments. Herit Sci. 2013;1:23. 
90. Cameron CA. Art. XIV-report on poisonous and adulterated confectionery. Dublin Q J Med Sci. 1871;51:150-5.

91. Cameron CA. Note on the operation in Dublin of the acts relating to adulteration. Analyst. 1885;10:175b-179.

92. Carlyle L. Historically accurate reconstructions of oil painters' materials: an overview of the hart project 2002-2005. In: Boon JJ, Ferreira ESB, editors. Report highlights mayerne programme. The Hague: NWO; 2006. p. 63-76.

93. Carlyle L, Witlox M. Historically Accurate Reconstructions of Artists' Oil Painting Materials. Tate Pap. 2007;7. https://www.tate.org.uk/research/ publications/tate-papers/07/historically-accurate-reconstructions-of-artis ts-oil-painting-materials. Accessed 29 Oct 2018.

94. Hendriks L, Hajdas I, Ferreira ESB, Scherrer NC, Zumbühl S, Küffner M, et al. Selective dating of paint components: Radiocarbon dating of lead white pigment. Radiocarbon. 2018. https://doi.org/10.1017/RDC.2018.101.

95. Abdi H, Williams LJ. Principal component analysis. Wiley Interdiscip Rev Comput Stat. 2010;2:433-59.

\section{Submit your manuscript to a SpringerOpen ${ }^{\circ}$ journal and benefit from:}

- Convenient online submission

- Rigorous peer review

- Open access: articles freely available online

- High visibility within the field

- Retaining the copyright to your article

Submit your next manuscript at springeropen.com 